U.S. Department of the Interior

Geological Survey

\title{
Experiments with SRO and GRF-array data
}

by

George L. Choy

Open-File Report $82-216$

1982

This report is preliminary and has not been reviewed for conformity with U.S. Geological Survey editorial standards. Any use of trade names is for descriptive purposes only and does not imply endorsement by the USGS. 


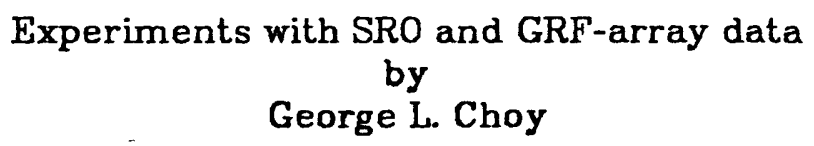

\section{INTRODUCTION}

This report summarizes the results of an on-site examination conducted in June 1981 of the instruments, facilities and data at the Graefenberg Seismological Observatory (GRF) located at Erlangen, Federal Republic of Germany. The first part of this paper provides a brief description of the array run by the observatory. The next part of the paper assesses the quality of the broadband data generated by the Wielandt seismometer by comparing its data with that generated by the SRO seismometer used by the USGS in its global network. GRF was an ideal site for making this comparison because at one of its stations an SRO is operating side-by-side with the Wielandt instrument.

Although the stations of the WWSSN and GDSN are globally distributed, they are sparsely spaced. This sparse spacing makes it difficult to evaluate the influence of the non-deterministic component of a body wave arrival on the overall pulse shape. An unstated assumption in many teleseismic body-wave inversions for source parameters and earth structure is that waveforms recorded at a single station are stable. That is, the waveform following a ray path with ray parameter $p$ and recorded at a distance $\Delta$ will be identical to a waveform that follows a slightly perturbed ray path having ray parameter $p+\delta p$ and recorded at an adjacent station at distance $\Delta+\delta \Delta$. This assumption is examined in the next part of this paper by using the GRF array. For teleseismic distances, the ray paths between the source and the stations of the array form a ray tube which subtends, for practical purposes, an infinitesimally small solid angle. Thus, the stability of a waveform can be determined by its coherence across the GRF array. Furthermore, the coherence of the waveform can be 
followed as a function of frequency because the recording instruments have a broadband response.

The final section of this paper describes potential research applications of the array. It could, for instance, be used to refine upper mantle structure in Europe because the distance between the array and many seismically active regions is near the cusp distance for many body phases. The broadband horizontal recorders in the array also provide the resolution for detecting anisotropy of S-waves.

\section{THE GRF ARRAY}

A brief description of the GRF array is given here because references to its characteristics are made throughout subsequent sections of the paper. A more detailed description can be found in Harjes and Seidl (1978).

The instrument used in the stations of the array is a Wielandt seismometer. Like the SRO seismometer, it operates on the force-balance principle. Its response characteristics, dynamic range and in a sense even its stability is a function of an electronic rather than mechanical system. It is physically about the same size as the SRO. However, the method of installation and some subsequent calibration procedures require that a three-component set of the instruments occupy a vault that is comparable in size to those of the WWSSN. This precludes its installation in a borehole. While the output of the SRO is proportional to acceleration from 0.02 to $1 \mathrm{~Hz}$, the Wielandt output is proportional to velocity from 0.05 to $5 \mathrm{~Hz}$. The broadband output from the Wielandt is recorded continuously with a sampling rate of 20 samples/sec.

The configuration of the GRF array is shown in Fig. 1 . The 13 stations which comprise the array are grouped into three subarrays to optimize telemetry. The central stations of the three arrays are designated as $\mathrm{A} 1, \mathrm{~B} 1$ and $\mathrm{C} 1$. Satellite stations are designated by the letter of the subarray and a number. 


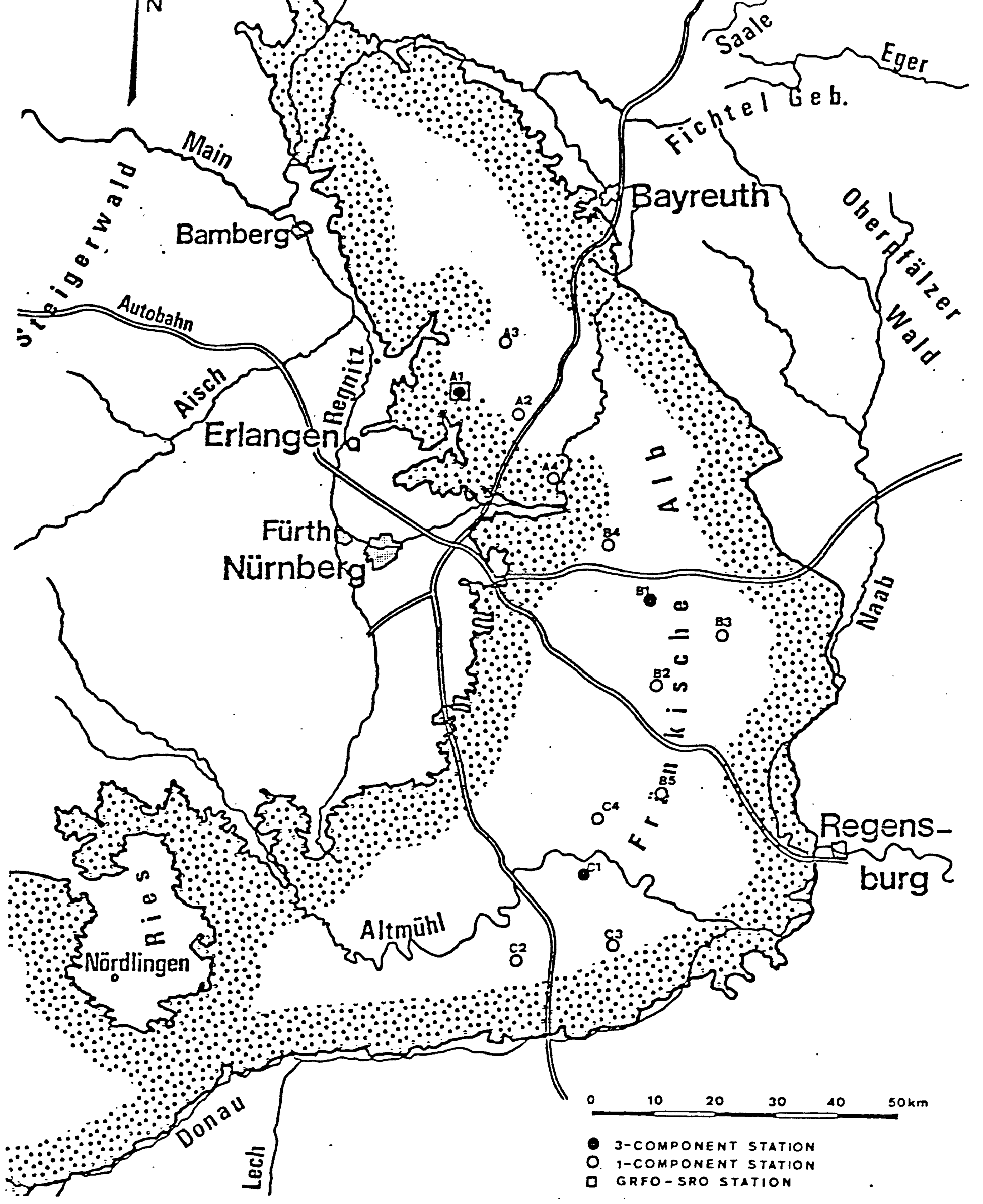

Figure 1. The configuration of the GRF array located in northern Bavaria. Each station is identified by a code consisting of one letter and one number. There are three subarrays identified by the letters A, B and C. All sites have one vertical sensor. The center station of each subarray records two horizontal channels as well. 
Each satellite has only a vertical sensor. The central stations have a threecomponent set of sensors. Station GRFO which consists of a three-component set of SRO sensors is located at the A1 station.

\section{COMPARISON OF SRO AND WIELANDT DATA}

In this section, the quality of data generated by the Wielandt and SRO instruments is compared. The facilities at GRF afford the most direct means of doing this because three-component sets of these instruments are operating side-by-side at the A1 station. In fact, the SRO at the A1 station is located in a borehole about $30 \mathrm{~m}$ laterally and $100 \mathrm{~m}$ vertically from the Wielandt seismometer. The Wielandt seismometer has a broadband amplitude response that is flat to velocity between $0.05-5 \mathrm{~Hz}$. Though the SRO output is also broadband, being flat to acceleration from 0.02 to $0.5 \mathrm{~Hz}$, it is not recorded. Instead, for the purpose of optimizing the detection of small signals, long-and short-period channels are recorded after the broadband signal is split and passed through different shaping filters. This precludes a straightforward comparison of spectral information in the intermediate-frequency range ( $\approx$ 0.08-1.0 Hz), although the broadband SRO signal can often be retrieved by suitable deconvolution techniques (Harvey and Choy, 1981). However, for this study, it is desirable to compare the data sets with the least processing of data. The GRF scientists, fortunately, were able to tap the broadband output from the SRO. A program was then developed that converted the SRO acceleration into velocity.

In the rest of this section data from seismic sources that were simultaneously recorded by the two instruments at the A1 station are compared. The magnitude $\left(\mathrm{m}_{b}\right)$ of the sources ranged from 5.4 to 6.7 in order to permit an evaluation of the data as a function of dynamic range and frequency. This is also the first time that broadband signals from the horizontal SRO 
components could be examined. (Only a long-period component is normally recorded for the horizontal channels at SRO stations.)

\section{Small earthquakes}

The P-waves as recorded by the two sets of instruments for a small $\left(m_{b} 5.4\right)$ earthquake in Tibet are shown in Fig. 2. The first set of records shows the three components of velocity from the Wielandt seismometer. The records in the second set are from the SRO instrument. Its broadband output has been shaped so that it is flat to velocity from $0.02-9 \mathrm{~Hz}$. The $P$-waves are for all practical purposes identical. The similarity extends even down to the coda and pre-signal noise. Some slight discrepancies seen as long-period riders probably arise from windowing effects. The low-frequency corner below which the amplitude response of the Wielandt instrument falls off is near $0.05 \mathrm{~Hz}$, whereas the processed SRO response falls off at $0.02 \mathrm{~Hz}$.

\section{Large earthquakes}

The three component set of records for the P-waves from the Eureka, California, event of November 8, 1980, are shown in Fig. 3. Relative to the event that was just discussed, this earthquake is enriched in long-period energy because it was so large $\left(m_{b} 6.7 ; M_{s} 7.2\right)$. As before, the waveforms recorded by the two sets of instruments are very similar. There are minor differences at the low frequencies which probably arise from the different corner that shapes the low-frequency end of the two velocity responses. Additional differences in the horizontal records are because the surface-installed Wielandt instruments are more subject to wind-generated noise and tilt than the SRO's, which are buried in a borehole.

\section{Low-frequency response}

The low-frequency response of the Wielandt seismometer can be evaluated by simulating an SRO long-period record from the Wielandt broadband record. 

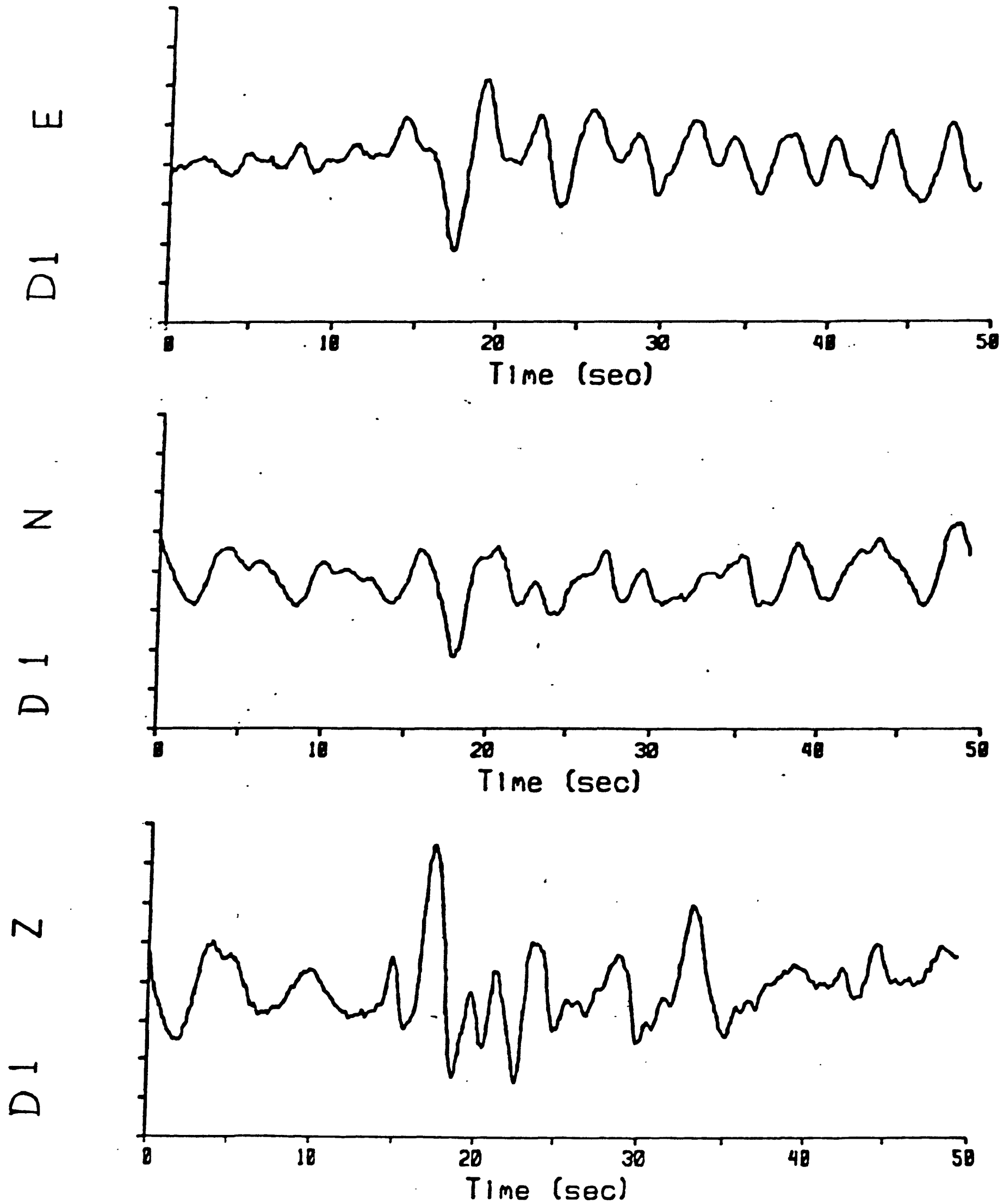

Figure 2b. The corresponding P-waves for the Tibet event as recorded at the SRO station, GRFO, which is also located at the A1 station. The broadband SRO channels are designated as "D1". For comparison with the Wielandt output, the broadband output from the SRO has been converted to velocity. 


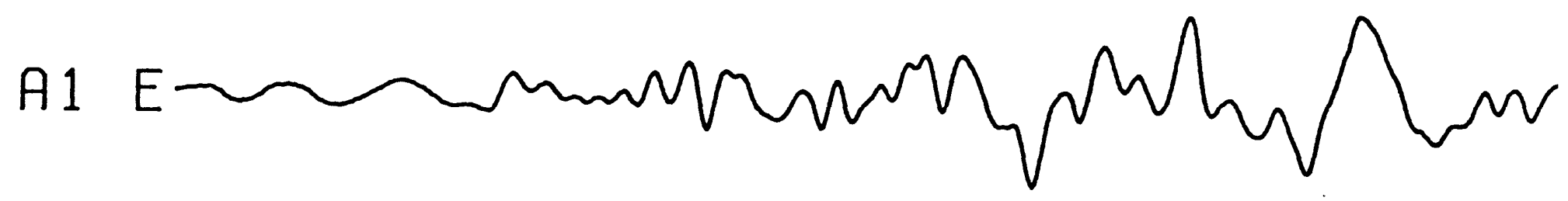

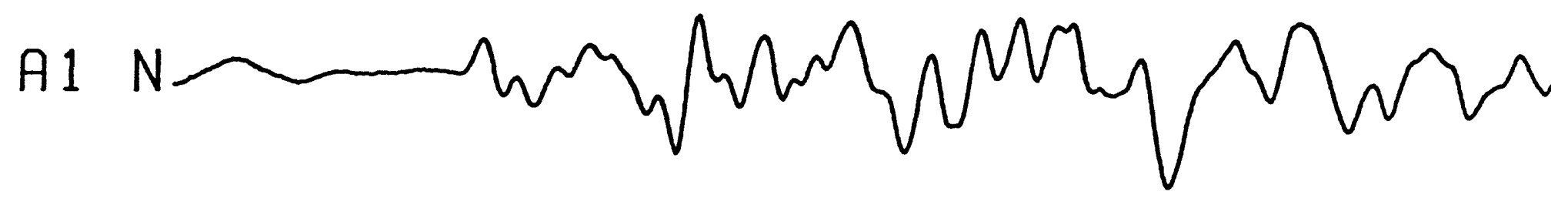

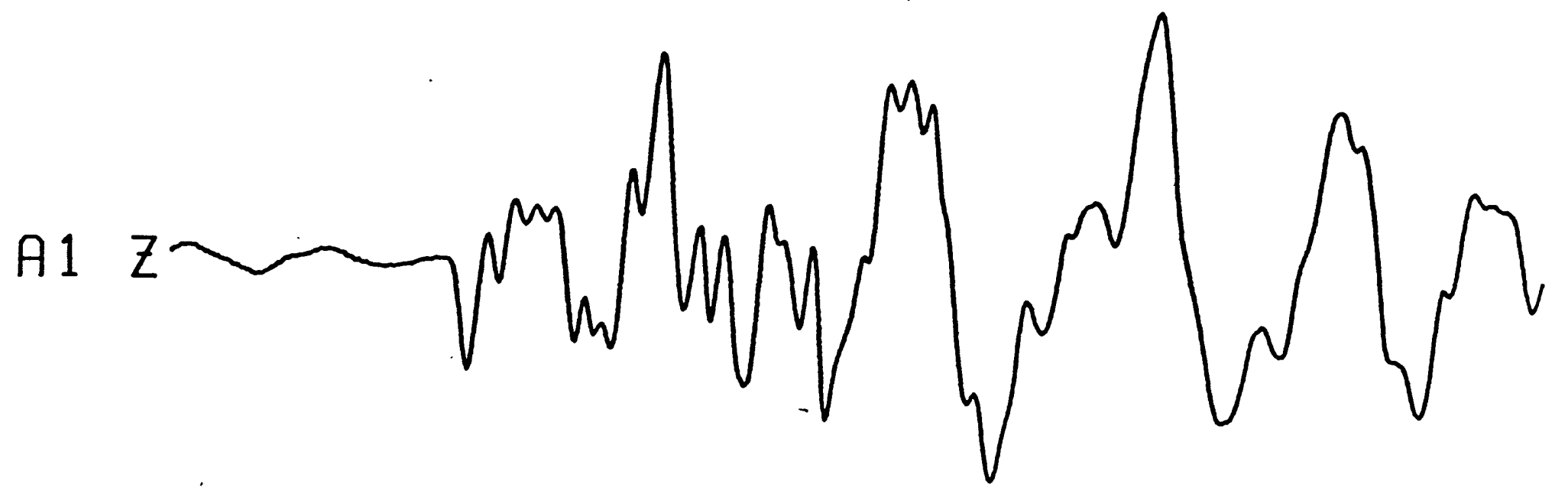

$10: 39: 40$

Figure 3a. Broadband P-wave section for the large Eureka, California, earthquake of November 8,1980 as recorded by the Wielandt sensors at the A1 station. The hypocentral parameters for this event are as follows: OT

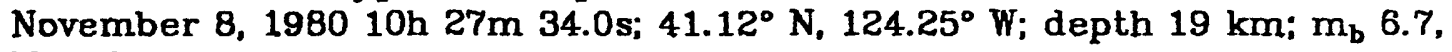
$\mathrm{M}_{S}$ 7.2. 
D1 E
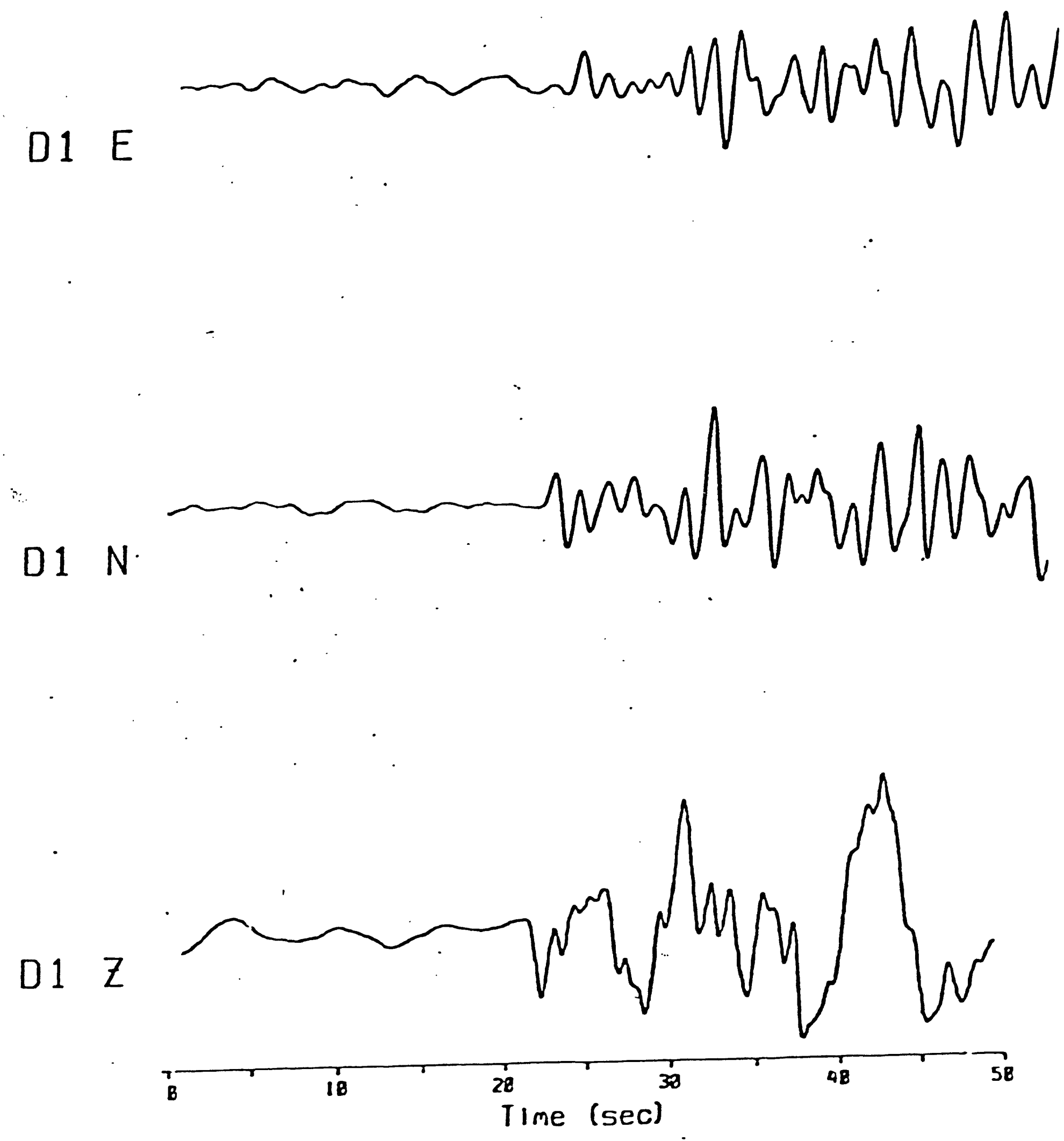

Figure 3b. The corresponding P-waves for the Eureka, California, event as recorded at the SRO station. For comparison with the Wielandt output, the SRO data has been converted to velocity. 
This effectively low-pass filters the Wielandt output. Such a comparison is shown in Fig. 4 for a surface-wave train. The actual and simulated SRO records agree almost exactly with regard to amplitude and dispersion. In general, however, this comparison is not as good for the horizontal seismometers. Because of their surface installation, the Wielandts are more sensitive to wind-generated microseisms. Though this may not be too significant at GRF, it could be a very important factor at other stations.

\section{High-frequency response}

The high-frequency response of the Wielandt seismometer can be evaluated by simulating the SRO short-period response from the Wielandt output. This effectively high-pass filters the data. Fig. 5 compares the P-wave from the Eureka, California, event that was recorded on the SRO short-period channel with the simulated short-period SRO record. They are practically identical although the Wielandt record appears to have slightly different high-frequency ( $\geqq 3 \mathrm{~Hz}$ ) noise which may be because its surface installation is more susceptible to cultural noise.

\section{Verification of broadband deconvolution}

The SRO long-period response has a twin- $T$ notch filter at $.166 \mathrm{~Hz}$ to minimize microseismic noise. Fortunately, the intermediate frequencies that are notched out can be retrieved from the short-period channel because of the high dynamic range of the recording system. A method for retrieving the original broadband ground displacement and velocity from the simultaneous deconvolution of the long- and short-period SRO channels has been described by Harvey and Choy (1981). As a direct broadband output was being recorded from the SRO instrument at station A1 during this comparison study, a simple verification could be made to see if the the output predicted by the deconvolution program is identical to the actual broadband output. The 

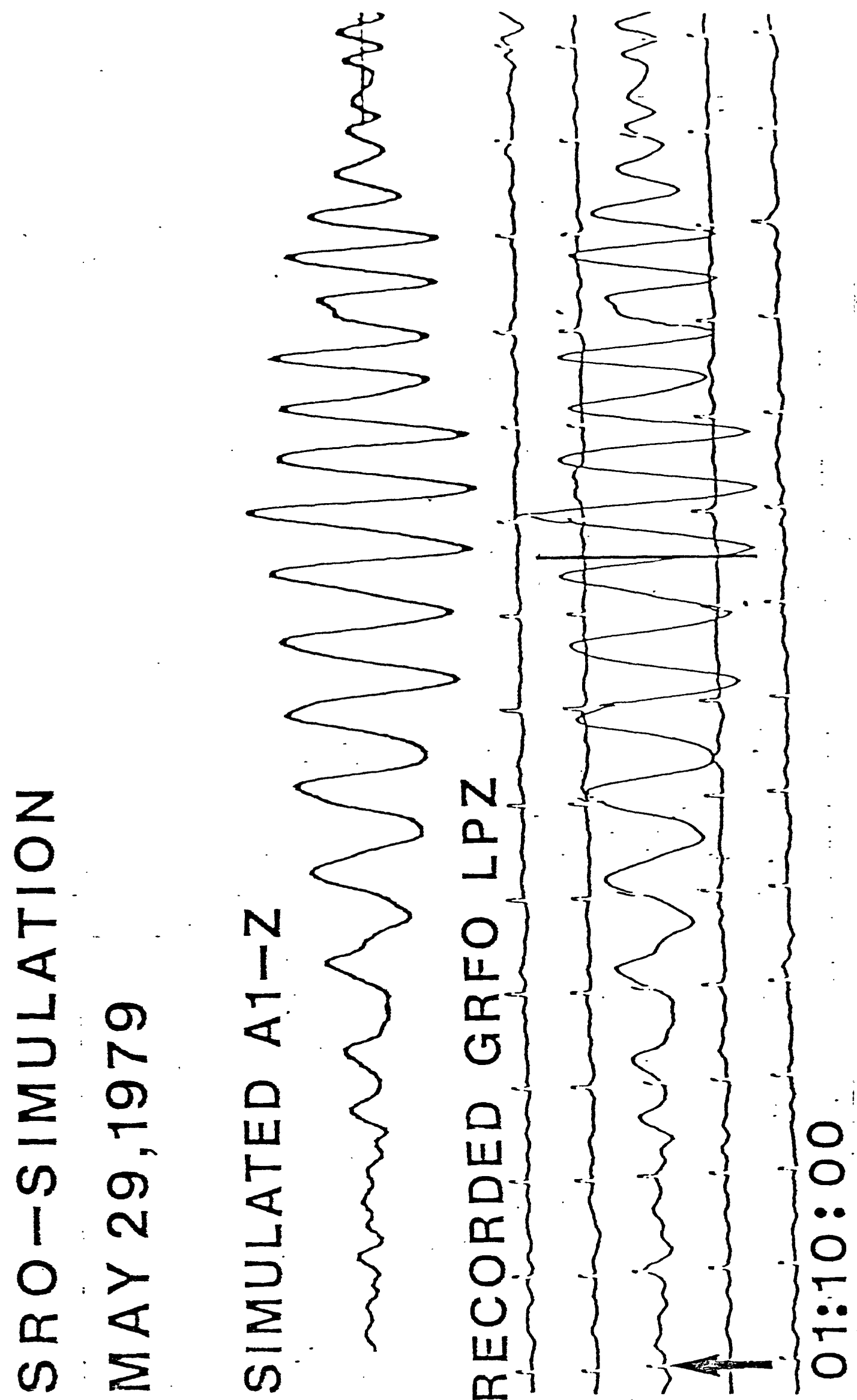

통 열 명 넝

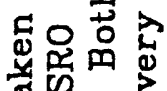
a ข 紧苛 过琞 ญ. 造 世 ज菏。 ه 䗆 넝옹용 대웜 ข

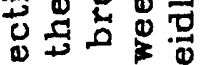

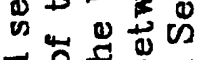
هั

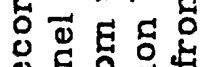
ఎ

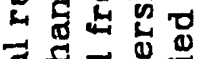
สี 可 ه

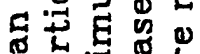

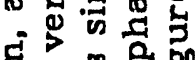
E क व ㅇํㅇ +艹

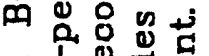
+ ob ๑ 동 늠

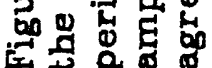





Figure 5. Top, an SRO short-period simulation using the Wielandt data for the P-wave of the Eureka, California, earthquake of November 8, 1980. Bottom, the actual data from the short-period channel of station GRFO. (Amplitudes of the records were not normalized.) 
broadband velocity for the P-wave of the Eureka, California, event that was obtained directly from the SRO seismometer is shown at the top of Fig. 6. The trace below it is the velocity obtained from the simultaneous deconvolution of the long- and short-period output of the SRO. The traces are identical.

\section{Conclusions}

In summary, the Wielandt and SRO instruments are comparable. Both are capable of broadband recording. The records from one instrument can be simulated from the records of the other. in the frequency range from $0.02-5 \mathrm{~Hz}$. However, because of the surface installation of the Wielandt instruments, the horizontal sensors are more sensitive to wind-generated noise at long periods and all three components may be more susceptible to cultural noise at high frequencies. However, some of the noise above $3 \mathrm{~Hz}$ could be instrumental in origin. The examples in this comparison study also point out the extreme accuracy in the calibration of these instruments. The validity of simultaneous deconvolution to reconstruct broadband records from long-and short-period channels of SRO data was also demonstrated.

In terms of analyzing earthquakes, there are three negative aspects about the SRO's at present. First, in order to optimize detection of small signals, the dynamic range for recording the motion of very large earthquakes has been sacrificed. An example is shown in Fig. 7. The first set of seismograms are the Wielandt recordings of the October 10 1980, El Asnam, Algeria, earthquake $\left(\mathrm{m}_{\mathrm{b}}\right.$ 6.5) at a distance of $15^{\circ}$. The corresponding records from the SRO are all clipped and useless. Second, while intermediate-frequency data can be retrieved by using suitable digital processing techniques, it is an additional awkward computational step, especially for very large earthquakes with long source-time functions. Finally, there are no short-period horizontal recordings . Yet the notch filter that shapes the response of the long-period horizontal 

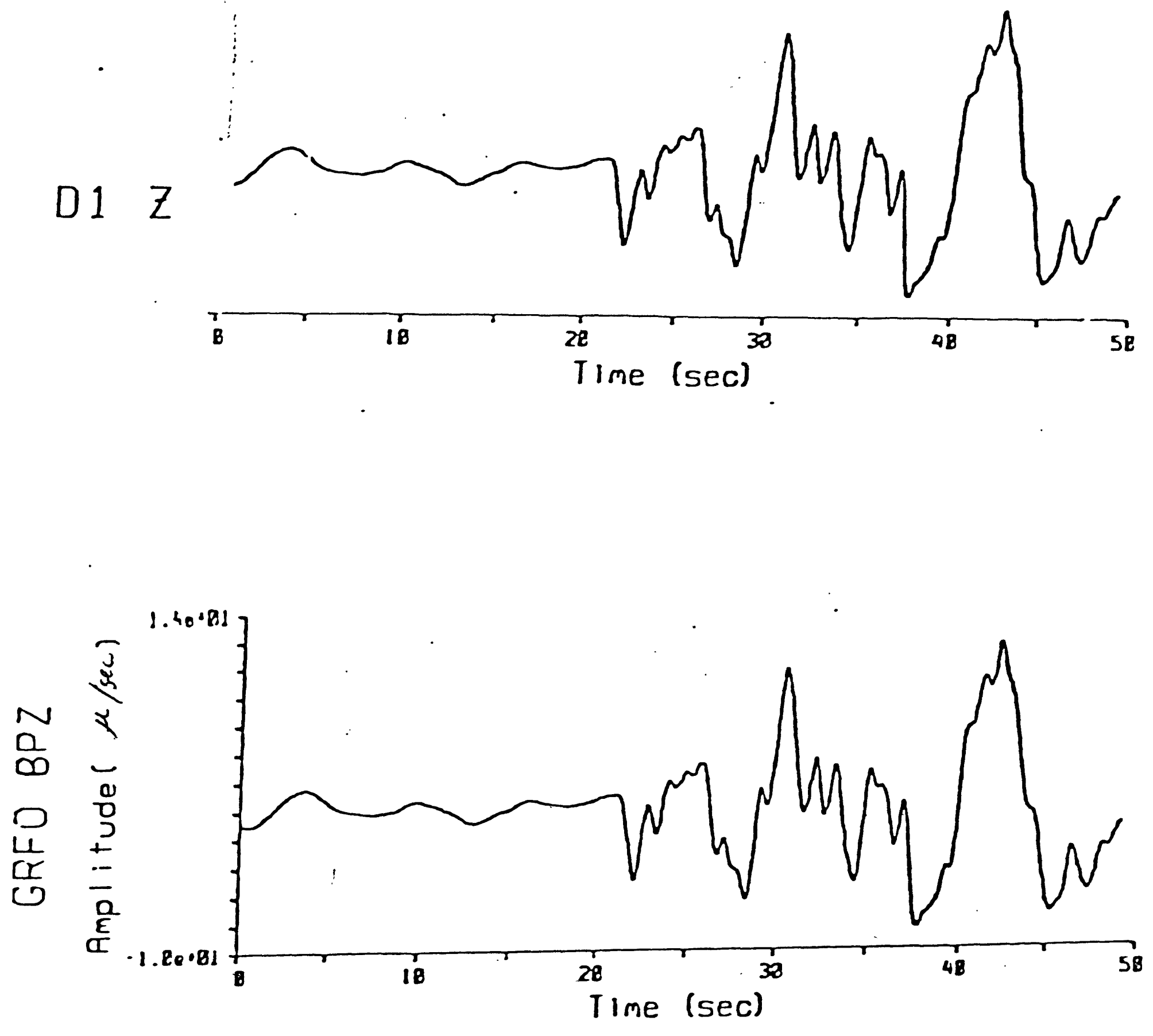

Figure 6. Top, the broadband P-wave velocity record from the Eureka, California, event obtained directly from the SRO seismometer. Bottom, the reconstructed broadband $P$-wave obtained by the simultaneous deconvolution of the long- and short-period output of the SRO. Amplitudes of the traces are normalized to the amplitude of the first trough in the Pwave. 

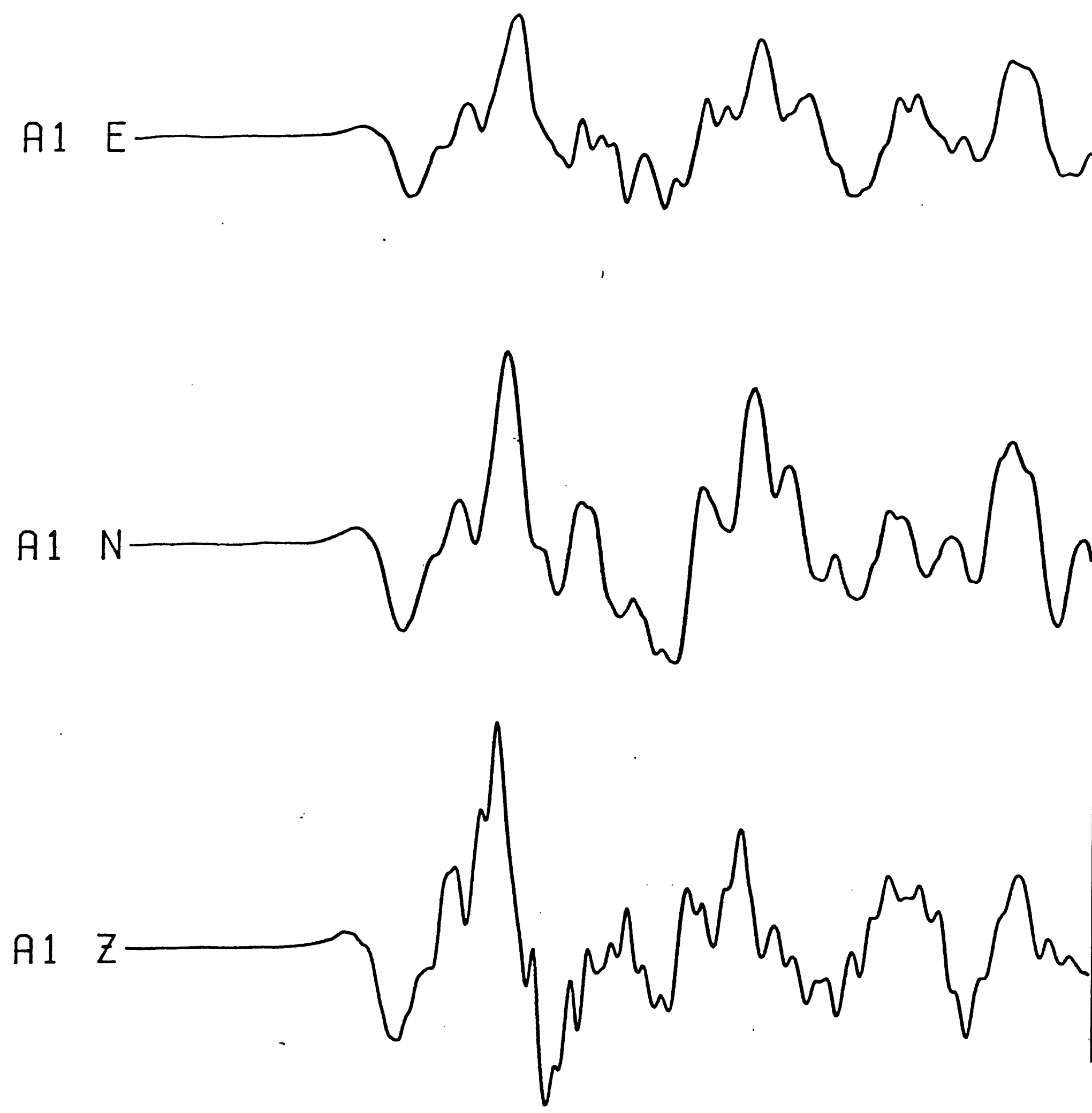

$12: 28: 50$

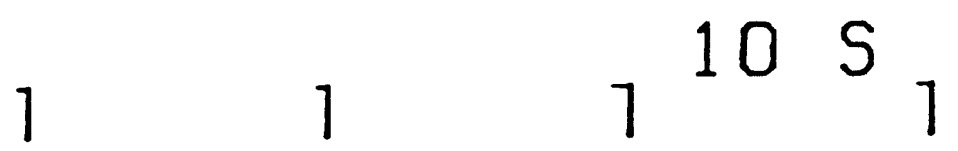

Figure 7a. P-waves recorded at the A1 station from the El Asnam, Algeria, earthquake of October 10, 1980. The hypocentral parameters were as follows: OT $12 \mathrm{~h} 25 \mathrm{~m} 23.58 ; 36.20^{\circ} \mathrm{N}, 1.35^{\circ} \mathrm{E}$; depth $10 \mathrm{~km} ; \mathrm{m}_{\mathrm{b}} 6.5, \mathrm{M}_{\mathrm{S}}$ 7.3. 

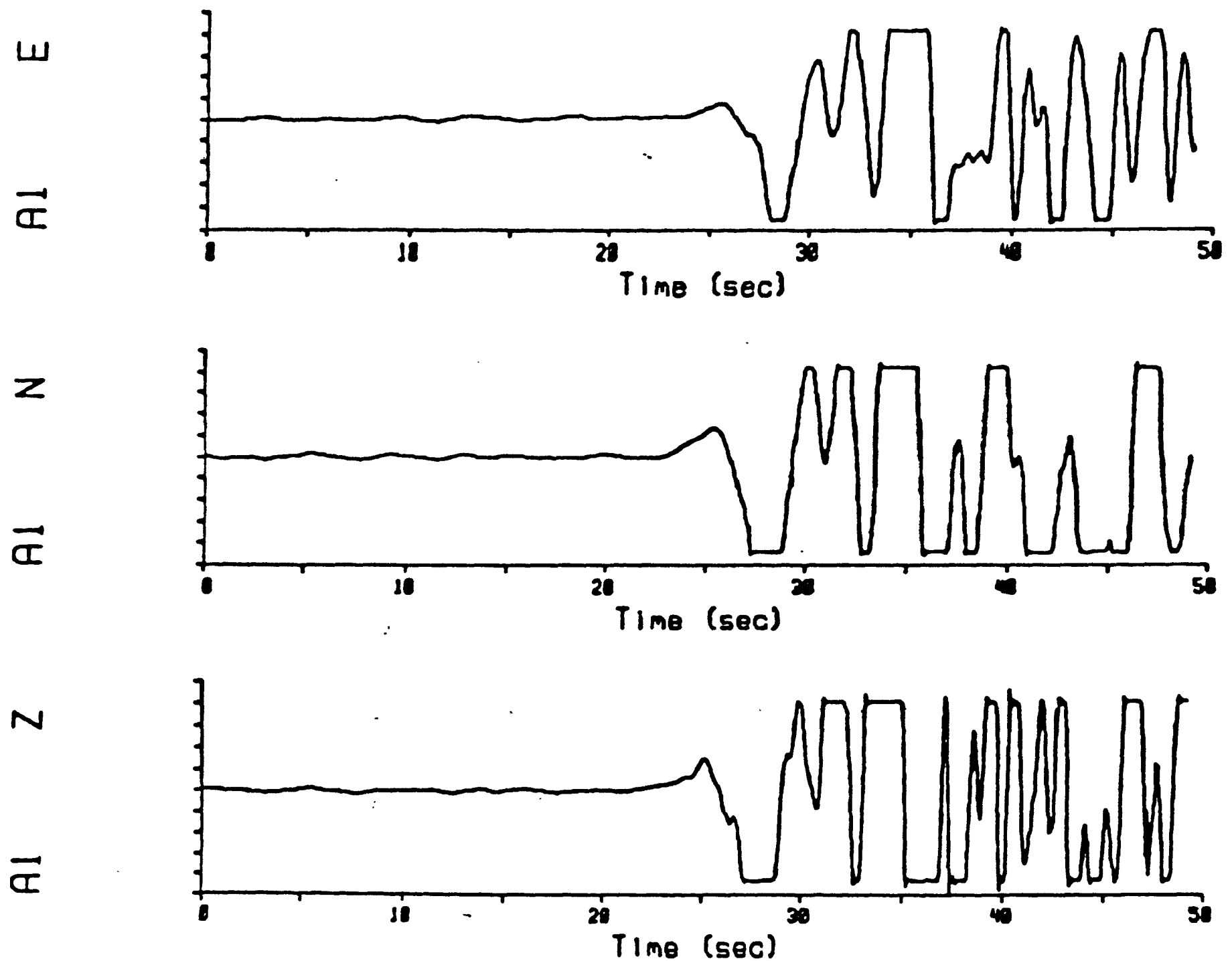

Figure 7b. Corresponding clipped $P$-waves as recorded by the SRO instruments. 
channels restricts the useful frequencies to less than $.08 \mathrm{~Hz}$. The higher frequencies that can provide strong constraints on structure or source inversion studies are completely lost because there are no short-period horizontal. recordings.

THE STABILITY OF PULSE SHAPES AS INFERRED BY THEIR COHERENCE ACROSS AN ARRAY

An underlying assumption in the inversion of far-field body waves for source mechanism or earth structure is that their pulse shapes can be deterministically modelled. Under certain conditions this assumption can be justified. One circumstance that has been widely exploited.is the inversion for source or structure using long-period body waves recorded by the WWSSN stations. These body waves contain wavelengths that are insensitive to all but the largest heterogeneities in the earth except the major discontinuities. A body wave with ray parameter $p$ that is recorded at a single station is assumed to be identical (with trivial corrections for geometrical spreading) to a body wave that arrives at an adjacent station by travelling a slightly perturbed propagation path with ray parameter $\mathrm{p}+\delta \mathrm{p}$. However, as advances in source theory now permit the computation of seismograms from increasingly complex and realistic rupture models, the frequency band that must be modeled has to be extended to intermediate and high frequencies ( $\approx 0.1-5 \mathrm{~Hz}$ ). The proper application of propagation theory to intermediate and high frequency data would also permit resolution of the fine structure in the earth and provide vital constraints on the relaxation constants that describe frequency dependent attenuation. In order to make use of these advances in source and propagation theory, it is necessary to determine the reliability of a waveform at a single station. This can be done by examining the frequency.threshold beyond which 
stochastic processes dominate a waveform.

The GRF array provides an excellent opportunity to address this problem. The instruments of the array record broadband data with a response that is flat to velocity in the frequency range $0.05-5 \mathrm{~Hz}$. By choosing appropriate seismic events, the effects of source complexity and near-source structure can be removed. Any differences in waveform across the array would have to come from structure beneath the station. If differences are observed, the next problem to address is whether the use of one waveform in an inversion for source or structure would produce a significantly different result than if the waveform from a station a few kilometers away were used.

\section{Earthquake sources}

In Fig. 8 are the waveforms across the GRF array for a deep earthquake that occurred in the Kuriles region. The epicentral distance to station A1 was $75.4^{\circ}$. The overall pulse shapes across the array are nearly identical. That is, the dominant features of any single waveform can be found in any other trace. These features are the initial peak, the subsequent triple trough and the last double-peak. Superimposed on the waveforms are high-frequency features, which appear to enhance the peaks and troughs on some records more than others. The greatest contrast occurs between the $\mathrm{B} 2$ and $\mathrm{A} 1$ traces. Of all the traces the B2 record seems to be the most depleted of high-frequency energy. On the other hand, the A1 record is very much enriched with high frequencies (as seen, for instance, in the sharpness of the second peak).

The origin of the high-frequency variation can be traced to upper-mantle or crustal heterogeneity beneath each station. This is because the cone of rays between source and array subtends an extremely small solid angle for teleseismic distances. Furthermore, for a deep earthquake, crustal and uppermantle effects (such as reflections from the underside of major structural 


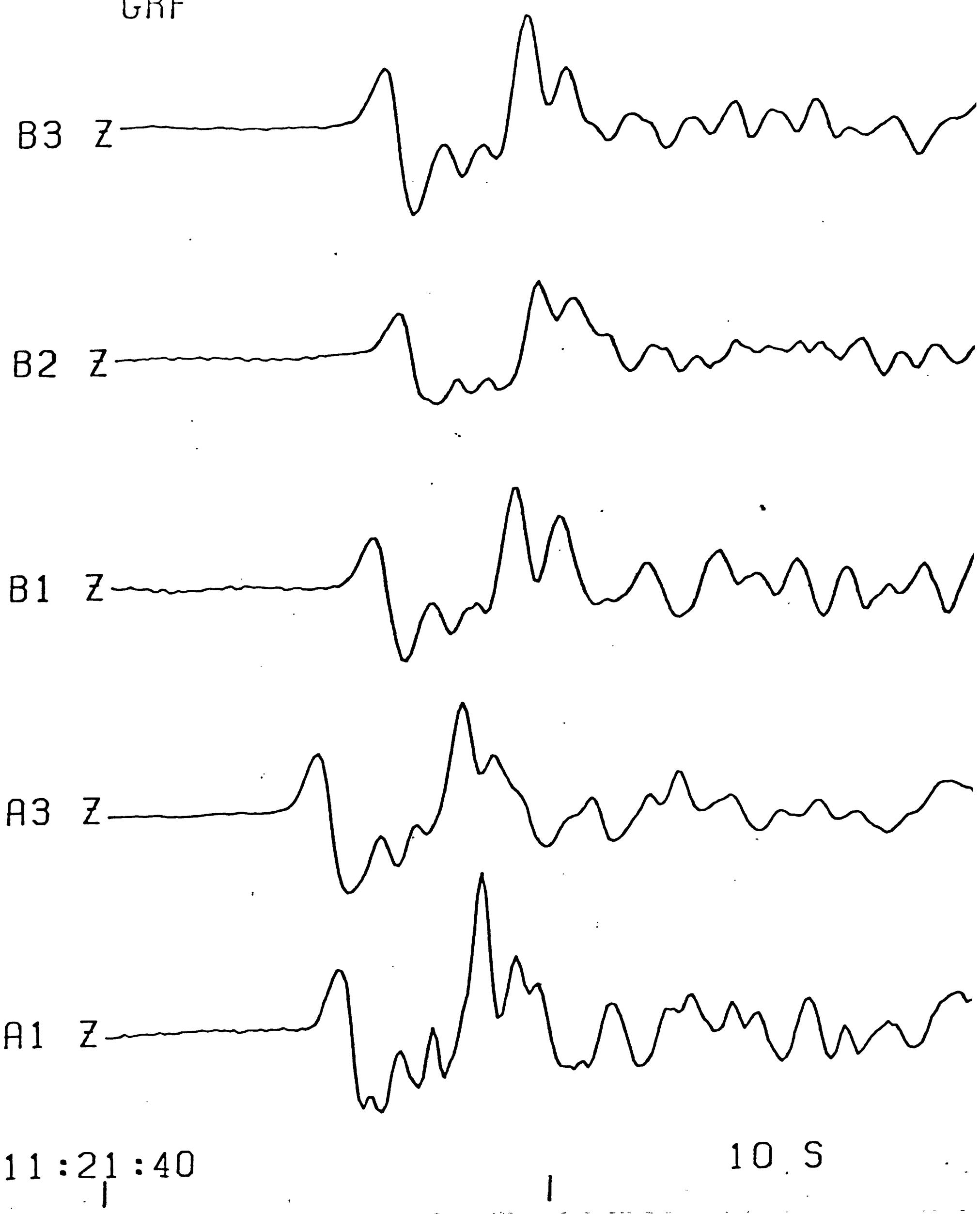

Figure 8. The broadband $\mathrm{P}$-wave velocity pulse shapes recorded by the array for an event in the Kuriles. Hypocentral parameters for this event were as follows: OT June $21,197811 \mathrm{~h} 10 \mathrm{~m} \mathrm{38.2s;} 48.3^{\circ} \mathrm{N}, 148.6^{\circ} \mathrm{E}$; depth $377 \mathrm{~km} ; \mathrm{m}_{\mathrm{b}}$ 5.9. The relative locations of the array stations are shown in Fig. 1. 
discontinuities) are less likely to arrive inside the time function of the source. Thus, the rays to each station in the array all incur the same effects during propagation near the source and in the lower mantle. Only on approaching each station of the array do the ray paths diverge significantly. Nevertheless, the arrival times of the peaks and troughs and the durations of the pulses of Fig. 8 can be followed across the suite of waveforms. This suggests that phase coherence is stronger than amplitude coherence. This observation is tested again later in Fig. 10.

The variation in the amplitude of high frequencies means that errors associated with an analysis in the time domain are likely to be smaller than an analysis in the frequency domain. An analysis in the frequency domain invariably discards phase information. In effect, an important degree of freedom is lost, which weakens the assumption that the signal is coherent. The variation in high-frequency energy might, for instance, change the apparent rolloff of amplitude with frequency, ultimately affecting estimates of corner frequency, radiated energy or corrections for attenuation.

A time-domain analysis was used by Choy and Boatwright (1981) to describe the rupture history for this earthquake. Fig. 9 summarizes the data used in their analysis. The solid lines are deconvolved velocity pulse shapes from the GDSN stations. The synthetics are given by the dashed lines. The GRF A1 record is inserted in the figure at the appropriate azimuth and takeoff angle with respect to the fault plane. Given the acceptable differences between the actual and synthetic records, one can see that any one of the records in the GRF array could have been used in the inversion without affecting the results.

The utility of time-domain analysis should be qualified, however. The analysis of Fig. 9 exploited broadband waveforms. Some analyses instead try to synthesize short-period body waves like those recorded by the WWSSN. Such 
Figure 9. Plotted about the focal sphere are the broadband deconvolved velocity pulse shapes (solid lines) and synthetic pulse shapes (dashed lines) for the suite of body waves recorded at GDSN stations from the Kuriles earthquake of June 21, 1978. Note that the fault plane and the takeoff directions of the body waves have been rotated so that the fault plane coincides with the plane of the stereonet. The downdip direction is indicated by the symbol in the center. The relative amplitude of the data to the synthetic is given by the number next to each body wave. The systematic variation of the waveform data from which rupture characteristics can be inferred is readily apparent. Starting clockwise from ANMO, observe that the P-waves are relatively compact (ANMO and CTAO). Successive P-waves at MAIO, CHTO and TATO develop a gradual doublet in the first trough and next peak. The $P P$ arrivals at ANMO and CHTO are even more strongly split. One of the waveforms from the GRF array is superimposed on the figure and its position on the focal sphere is indicated. (Figure modified from Choy and Boatwright, 1981.) 


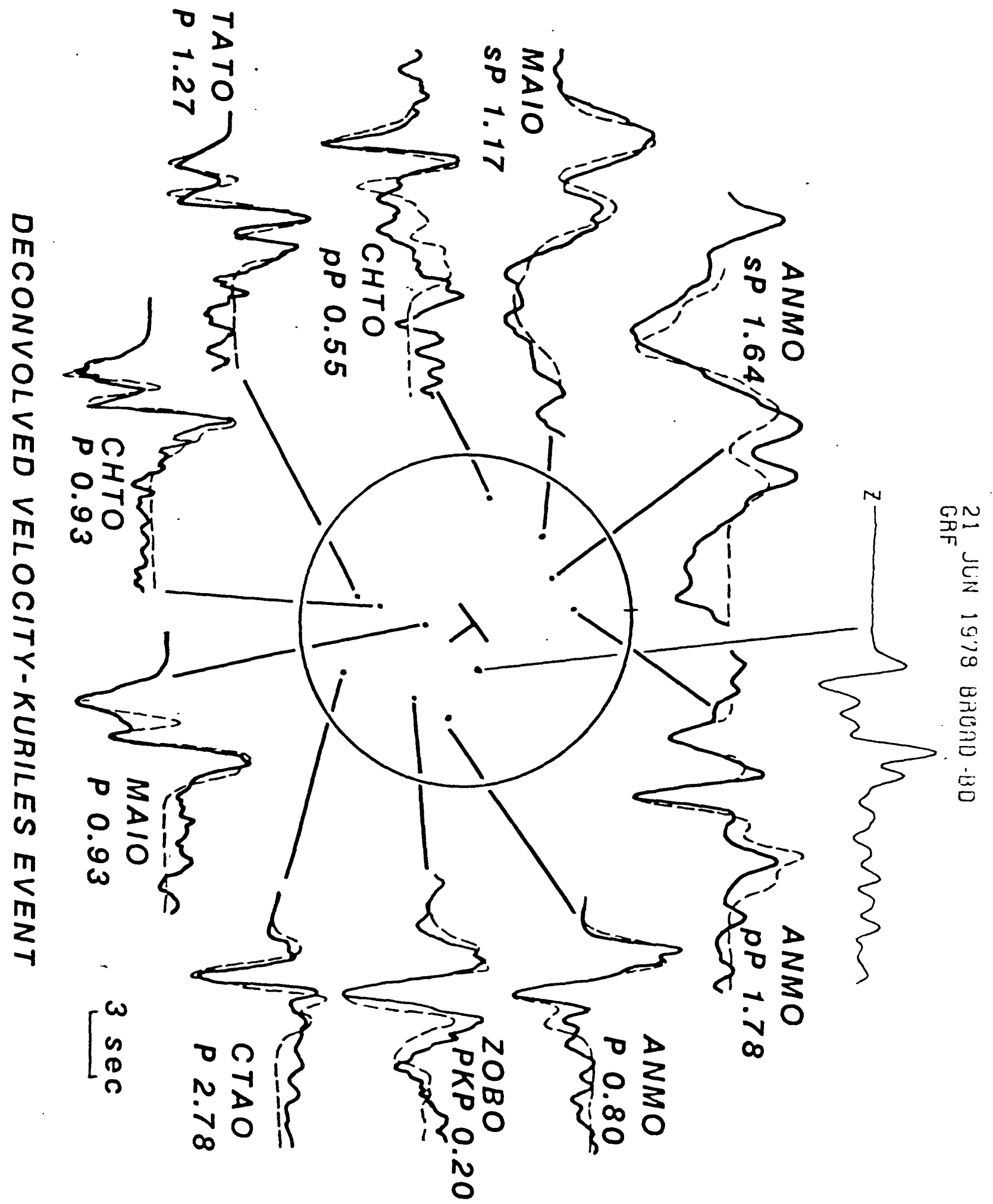


records are effectively narrowband filtered and de-emphasize coherent data in the intermediate frequency range. Thus, the resulting waveforms are more sensitive to station structure. Fig. 10 simulates the SRO short-period response using the traces of Fig. 8. The variation is very pronounced across the array. The relative height of peak 3 to peak 4, for instance, completely reverses character between stations B2 (where peak 4 is almost non-existent) and B1. Peak 5 would appear significant in the A1 record (being almost the same height at peak 3). But its amplitude is comparable to the coda noise in the B3 record. The amplitude and complexity of peak 2 relative to peak 1 also varies with station.

The superposition of all of the simulated traces is shown in Fig. 11. A substantial portion of amplitude space is covered by the waveform variations. However, the occurrences of the peaks and troughs are coherent. This is consistent with the observation that phase coherence is stronger than amplitude coherence. Nevertheless, exactly what can be modelled in the time- or frequency-domains with short-period (i.e., narrowband, high-frequency) data is subject to question.

\section{Explosive sources}

The variability of waveforms across the array is greater for an explosive source than for earthquakes. An example is the explosion that occurred in Eastern Kazakh on October 12,1980 . The distance to station $A 1$ is $42^{\circ}$. The P-waves across the array are shown in Fig. 12. Each pulse shape appears to be substantially different from the others. In particular the amplitude ratio of the first two peaks varies widely even though the aperture of the array is less than 1 degree. The complexity of the coda behind the second peak also changes drastically from station to station. The reason for the variations is that explosions are much more enriched with high frequencies than intermediate or 


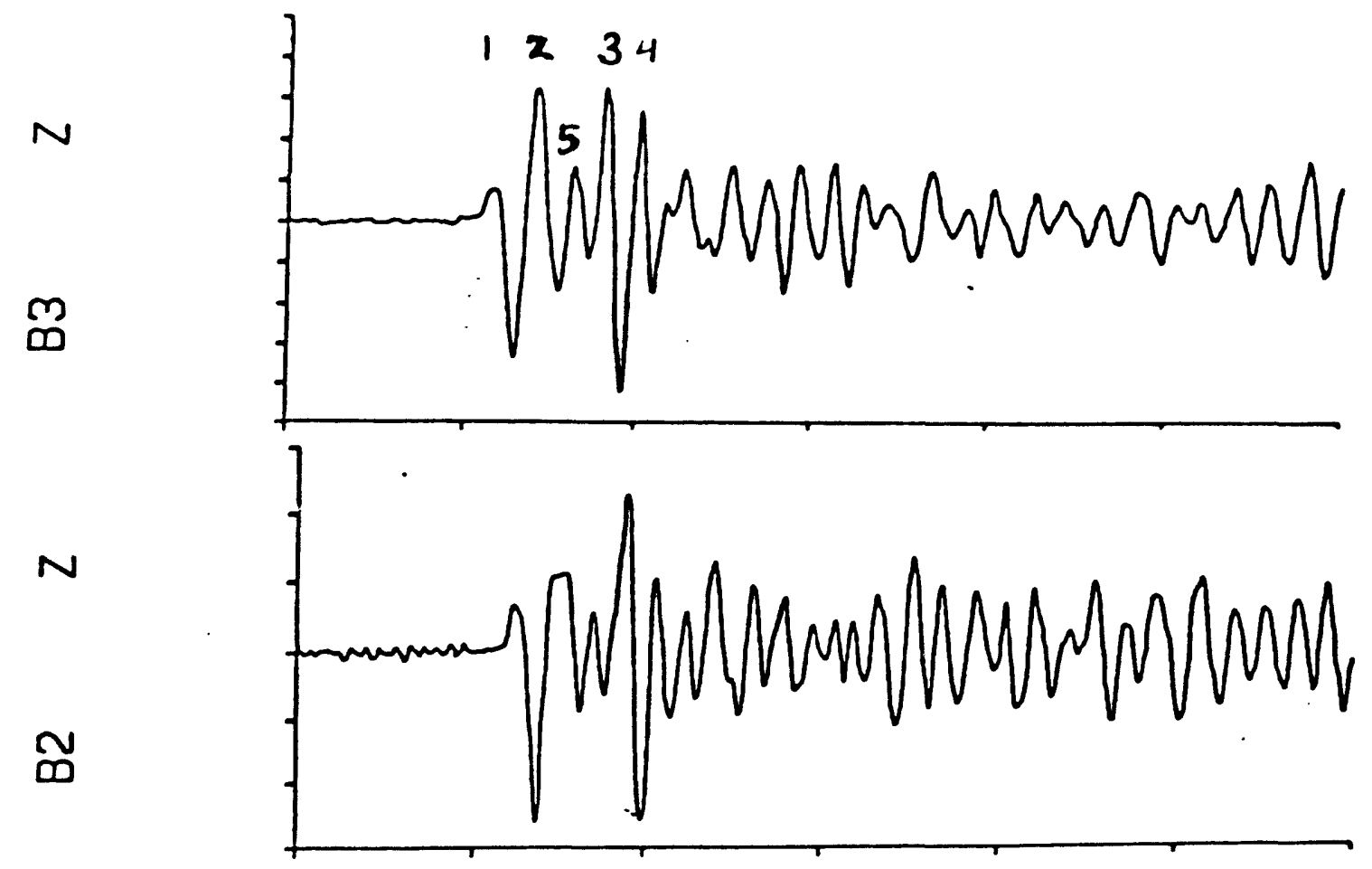

$N$

$\overrightarrow{0}$

N

๓

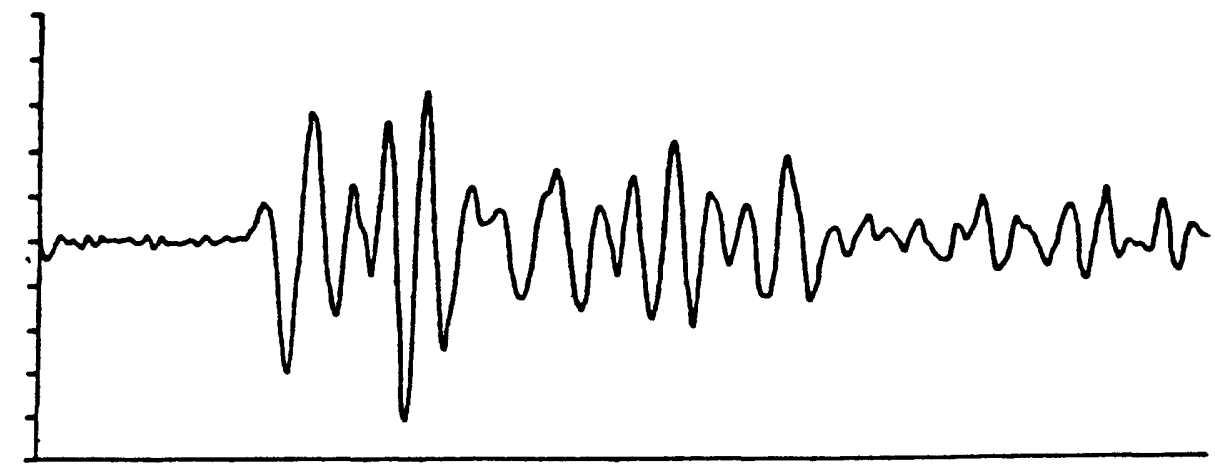

$N$

$\vec{\pi}$
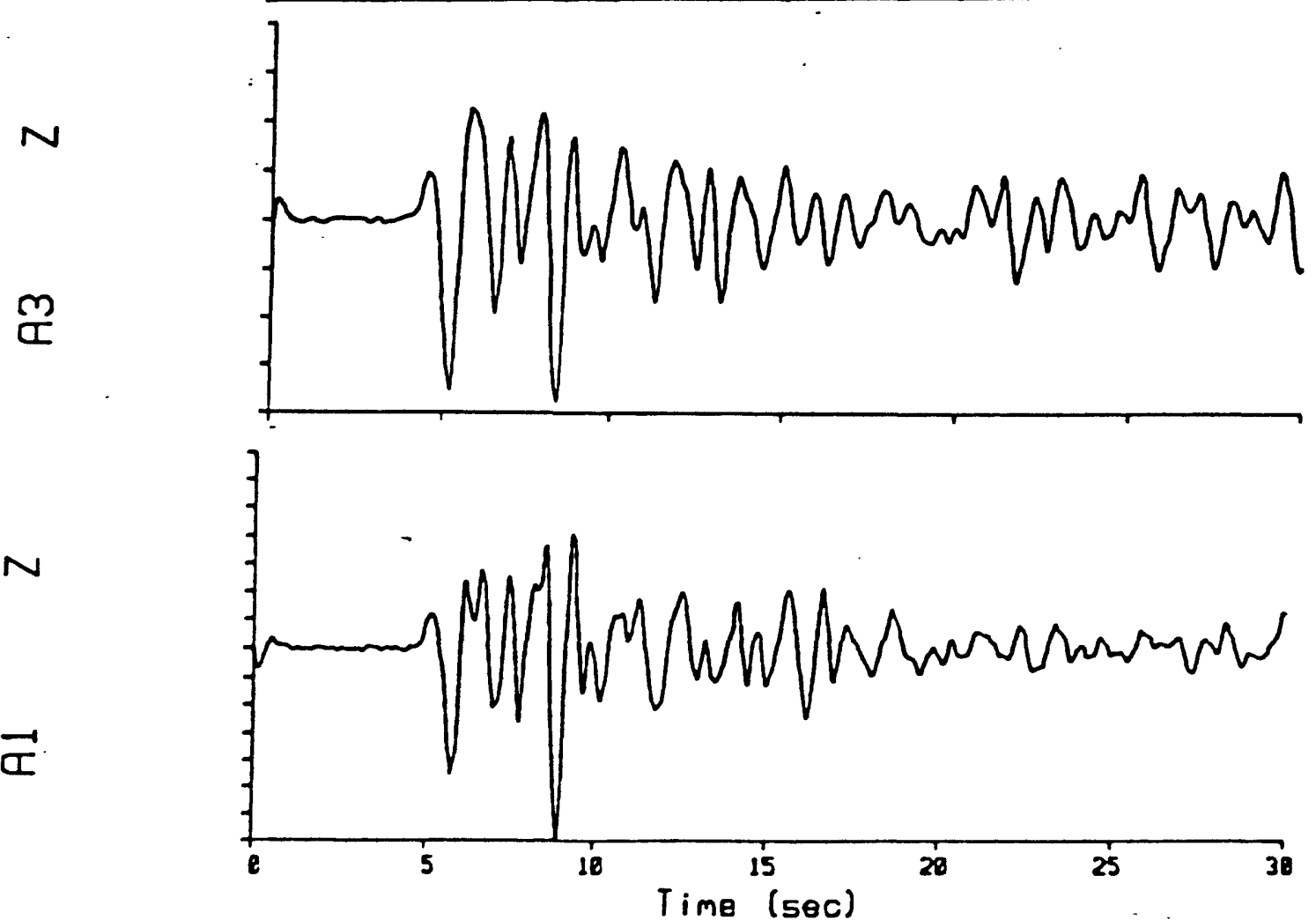

Figure 10. The broadband traces of Fig. 8 have been processed to simulate short-period SRO records. Note that the short-period records are essentially narrowband data with a peak response near $1 \mathrm{~Hz}$. 

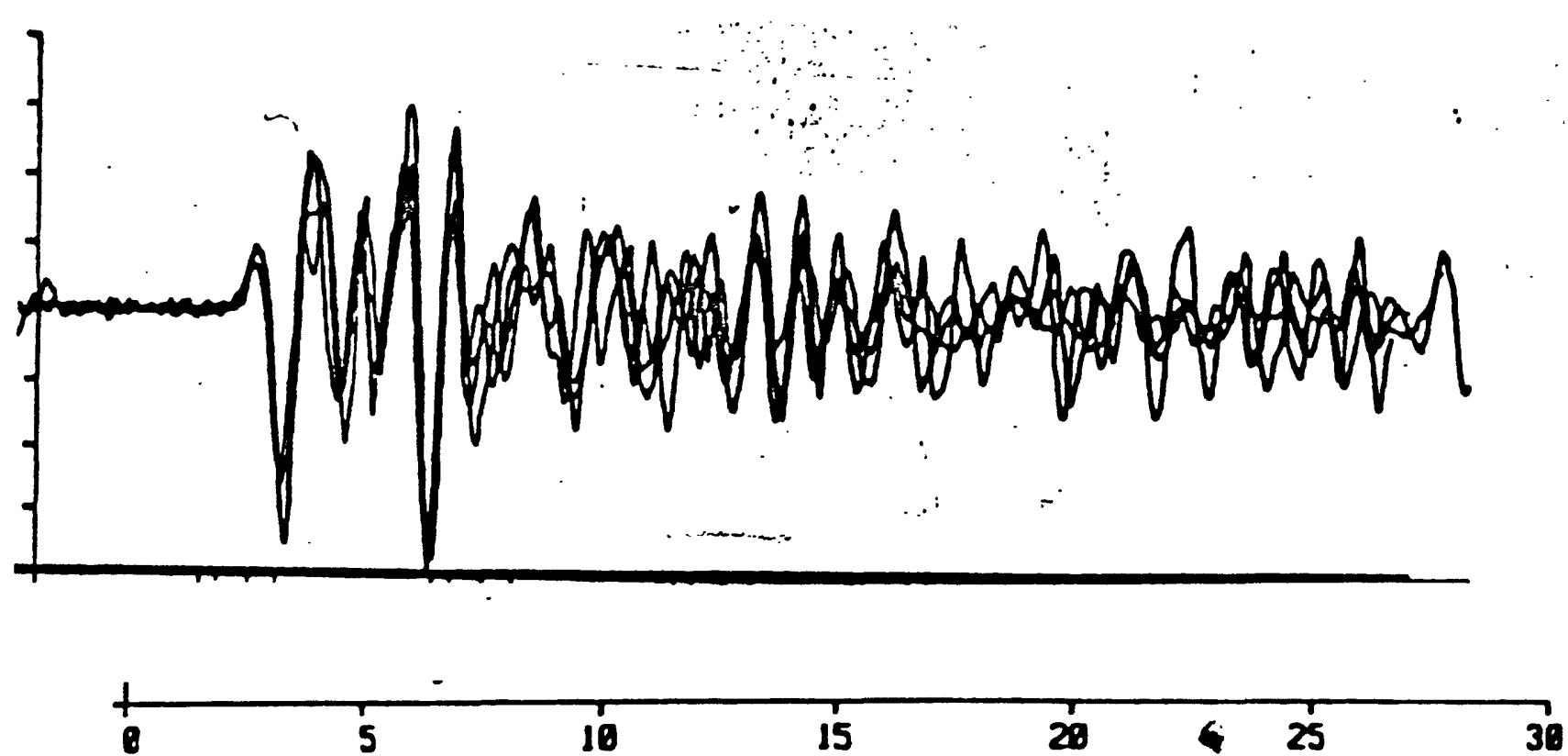

$(s . c . s)$

Figure 11. Superposition of the traces of Fig. 10 after correction for time delay at each station. Though amplitudes of the peaks and troughs of the pulses can vary considerably, their arrivals and durations are coherent. This suggests that phase coherence is stronger than amplitude coherence. 


$$
\text { (12) }
$$

Figure 12. Broadband P-wave velocity waveforms at the GRF array from the Eastern Kazakh explosion of October 12, 1980. The hypocentral parameters for this event from the are as follows: OT 03h $34 \mathrm{~m} \mathrm{34.1s;} 49.96^{\circ} \mathrm{N}, 79.08^{\circ} \mathrm{E}$; depth $0 \mathrm{~km} ; \mathrm{m}_{\mathrm{b}}$ 5.9, $\mathrm{M}_{\mathrm{S}}$ 4.3. The epicentral distance to the $\mathrm{A} 1$ station is $42^{\circ}$. 
low frequencies. Note, for instance, that the duration of the $\mathrm{P}$-wave pulse shape for the deep earthquake was about 6 seconds, while the duration of the explosion is no more than 3 seconds. Furthermore, the sharp peaks in the explosion Pwaves indicate the presence of energy higher than $2 \mathrm{~Hz}$. The explosive source also differs from the earthquake that was previously discussed by having occurred near the surface. Consequently, some of the variation in waveform may be the result of explosion-related phenomena (e.g., slapdown) as well as structure near the source. However, these effects are probably second-order relative to station effects. As before, it may be reasoned that, due to the tiny solid angle subtended by rays between the source and the substations of the GRF array, the greatest difference in the ray paths to the stations of the array occurs just beneath the stations. Thus, most of the variations in waveform are probably the result of the sensitivity of high frequencies to heterogeneities beneath a station.

The observation that explosion waveforms can vary drastically among stations that are separated by only a fraction of a degree has somber implications for their parameterization. It appears that station structure has a greater influence on waveform than can be described by any average spherically symmetric earth model. Parameters estimated in either the time or frequency domains will not be able to avoid large error bounds, depending on the degree of lateral heterogeneity in the vicinity of a particular station. The strong variability of a waveform also makes difficult the use of raw short-period waveforms as "master" events designed to identify events from a particular site. However, it would be possible to band-pass the waveforms until those frequencies that are sensitive to structure have been filtered out. It is also futile to try to estimate a receiver operator with high frequency content, even for a small solid angle of azimuth and takeoff angle, because slight changes in 
epicenter can cause significant changes in the interaction of high frequencies with receiver structure.

\section{Conclusions}

The stability of a pulse shape as inferred by an examination of its coherence across an array is frequency dependent. Qualitatively it can be said that higher frequencies are more sensitive to station structure than are the lower frequencies. Phase coherence appears much stronger than amplitude coherence. It may be that the various impedances and rough topography of the heterogeneity underneath a station affects amplitudes (by scattering, reflection and transmission) to first order but shifts phase only to second order. Because the effect of structure may vary from station to station, we have made no attempt yet to quantify the frequency threshold beyond which stochastic processes become dominant. It is clear, however, that the usefulness of parameters based on waveforms depends on how far the corner frequency of an event is removed from the frequency band at which incoherent phenomena dominate.

\section{OTHER RESEARCH APPLICATIONS}

The small aperture of the GRF array combined with its broadband recording capability makes the array suitable for a number of seismic investigations. Some of these topics are described briefly below. In some instances, preliminary results are also described.

Upper mantle structure

Body waves recorded by a coarsely spaced array (e.g., the WWSSN or the GDSN) can be modelled in order to derive the gross structure of the earth. However, the derivation of the fine structure of the earth requires the modelling of rapid changes in waveform that occur in the vicinity of certain critical distances. For instance, drastic changes in waveform can occur over the 
aperture of the GRF array if the distance to the source is close to a cusp. Fig. 13 shows an example using the P-waveforms from the Novaya Zemlya explosion of August 10, 1978. The distance between the A1 station and the epicenter is 30.25. A major change in waveform occurs between the A and B subarrays because at this distance all of their ray paths have turning points in the vicinity of the $670-\mathrm{km}$ discontinuity. Minute changes in ray parameter can cause a ray path to sample significantly different earth structure. For example, Fig. 14a shows synthetic displacement pulses for the $P$-wave interaction with uppermantle discontinuities for the PEM-C earth model. The last two synthetics were computed for distances of 29.1 and $29.6^{\circ}$. Although the synthetics were computed for a point source with no free surface reflections, their complexity is indicative of the propagation effects seen in the actual records across the array, which is about $30.25^{\circ}$ from the source. The strong differences between the waveforms are further emphasized in the corresponding velocity records (Fig. 14b). These waveform changes are predicted even though the station separation of the synthetic records is only $0.5^{\circ}$. The separation of some of the array stations is much less than that.

\section{Core structure}

The GRF array is also near the PKP-C cusp for events occurring in the New Hebrides region. The waveforms from an event in this region $\left(\Delta=145^{\circ}\right)$ are shown in Fig. 15. The cause of the variation across the array of the middle phase, PKPBC, can be found in the travel-time branches of PKP (Fig. 16). It results from the interference of PKP-BC with PKP-CD. The resolution of the cusp distance that can be obtained by modelling short-period and broadband waveforms from the GDSN is better than $2^{\circ}$ (Choy and Cormier, 1981). Using this array data the cusp distance could be refined to better than $0.3^{\circ}$. 


$$
\begin{aligned}
& 81 \text { z- }
\end{aligned}
$$

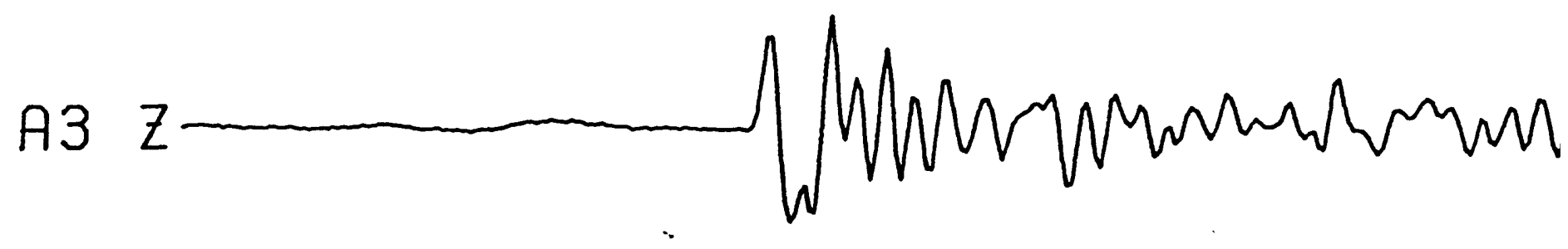
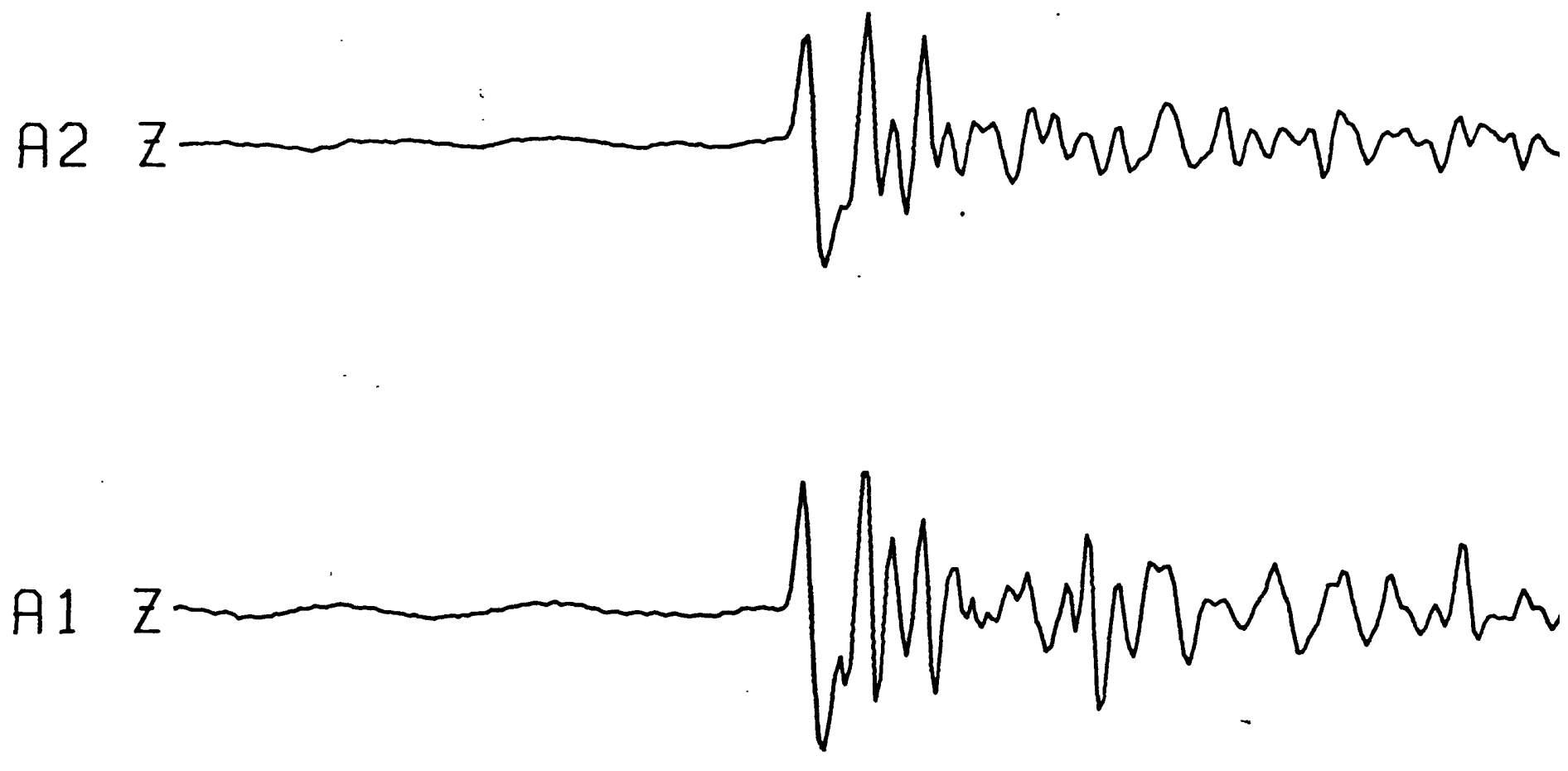

$8: 6: 0$

Figure 13. Broadband P-wave velocity waveforms across the GRF array from the Novaya Zemlya explosion of August 10, 1980. The hypocentral parameters are as follows: OT $07 \mathrm{~h} 59 \mathrm{~m} 57.7 \mathrm{~s}$; $73.34^{\circ} \mathrm{N}, 54.79^{\circ} \mathrm{E}$; depth OG km; $m_{b}$ 5.9. $\mathrm{Ms}_{\mathrm{s}} 4.3$. 


\section{PEM-C M(T)}

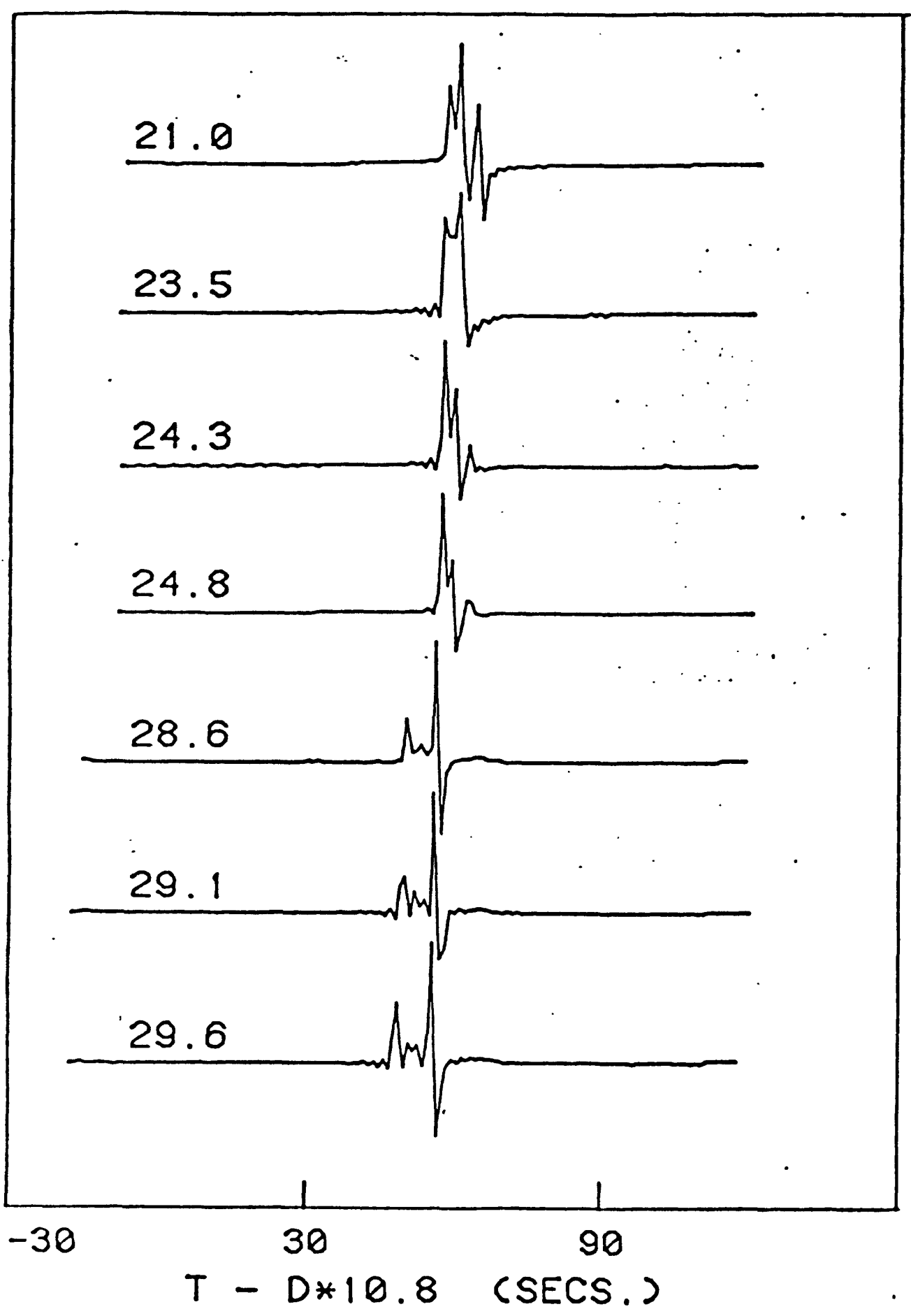

Figure 14a. P-wave displacement responses between $20-30^{\circ}$ predicted by the PEM-C earth model with the SL8 attenuation model for a point source at the earth's surface. Numbers next to each trace indicate distance. In this distance range, the $P$-waves are shaped by the interference pattern of travel-time triplications associated with upper-mantle discontinuities. (Figure modified from Cormier and Choy, 1981.) 


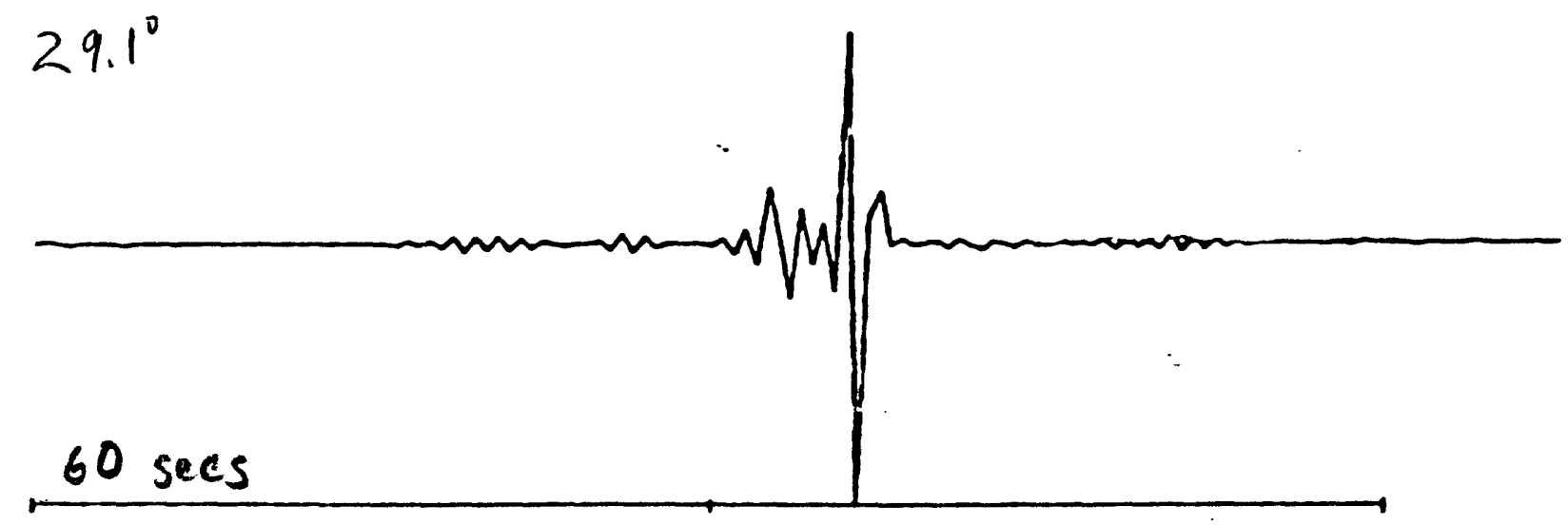

$29.6^{\circ}$

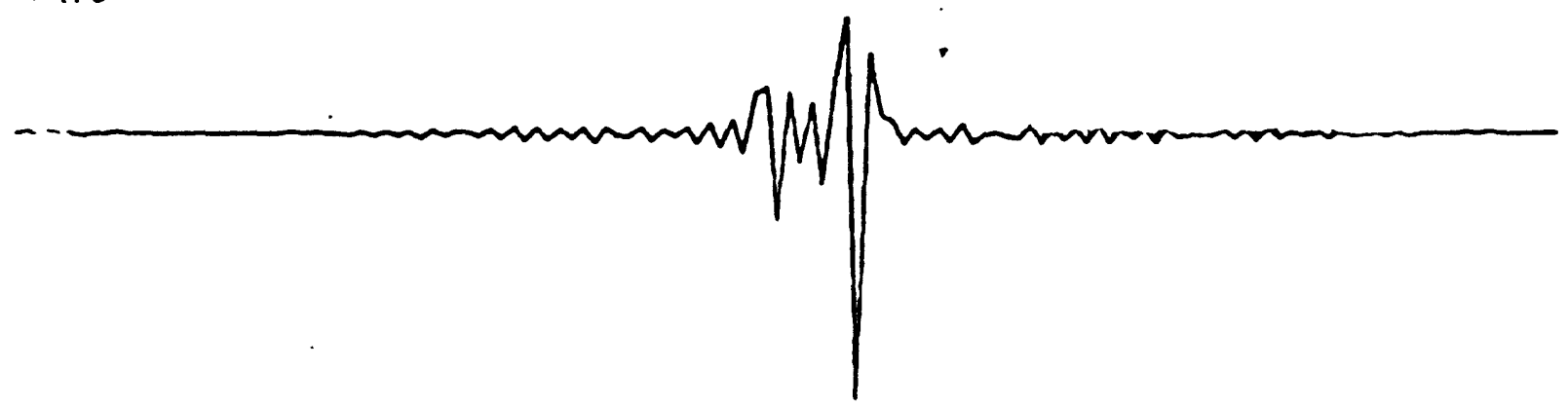

Figure 14b. The velocity records corresponding to traces in Fig. 14a that were computed for distances $29.1^{\circ}$ and $29.6^{\circ}$. (The small-amplitude ripples in the traces at the Nyquist frequency of $0.5 \mathrm{~Hz}$ are an artifact of the method of waveform synthesis. The actual Wielandt output has its Nyquist frequency at $10 \mathrm{~Hz}$.) 
20 JUN 1981 BROAD-BD

GRF

B5 $Z$ (

$B 4 \mathrm{Z}$

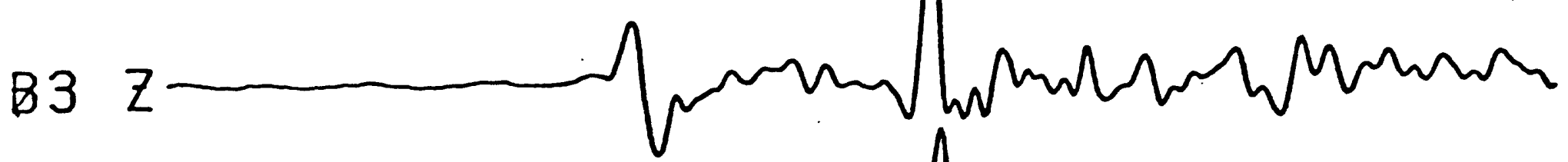

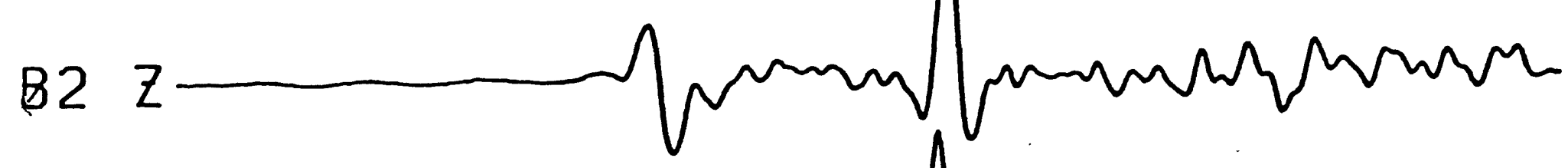

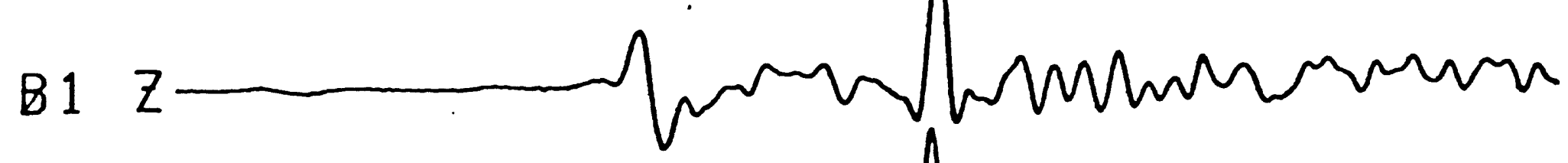

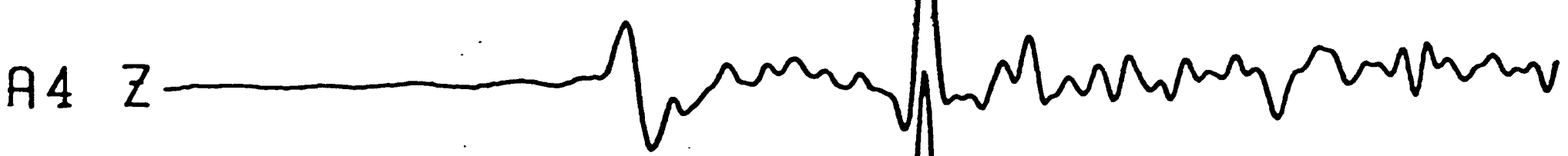

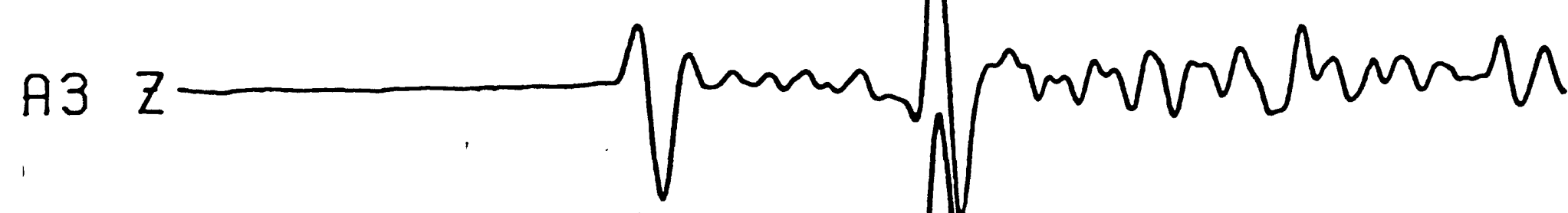

A1 $2-\overbrace{1}$ $4: 29: 20$

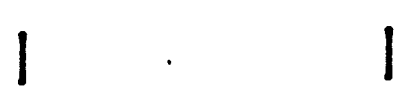

$10 \mathrm{~S}$

Figure 15. Broadband velocity waveforms of PKP body waves at the GRF stations. The event is from the Vanuatu Islands. The hypocentral

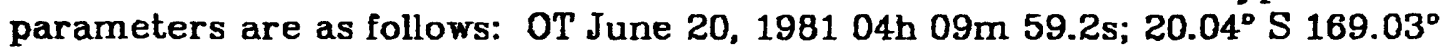

E; depth $32 \mathrm{~km} ; m_{b} 5.5, M_{S} 5.5$. The epicentral distance to the $A 1$ stations is 


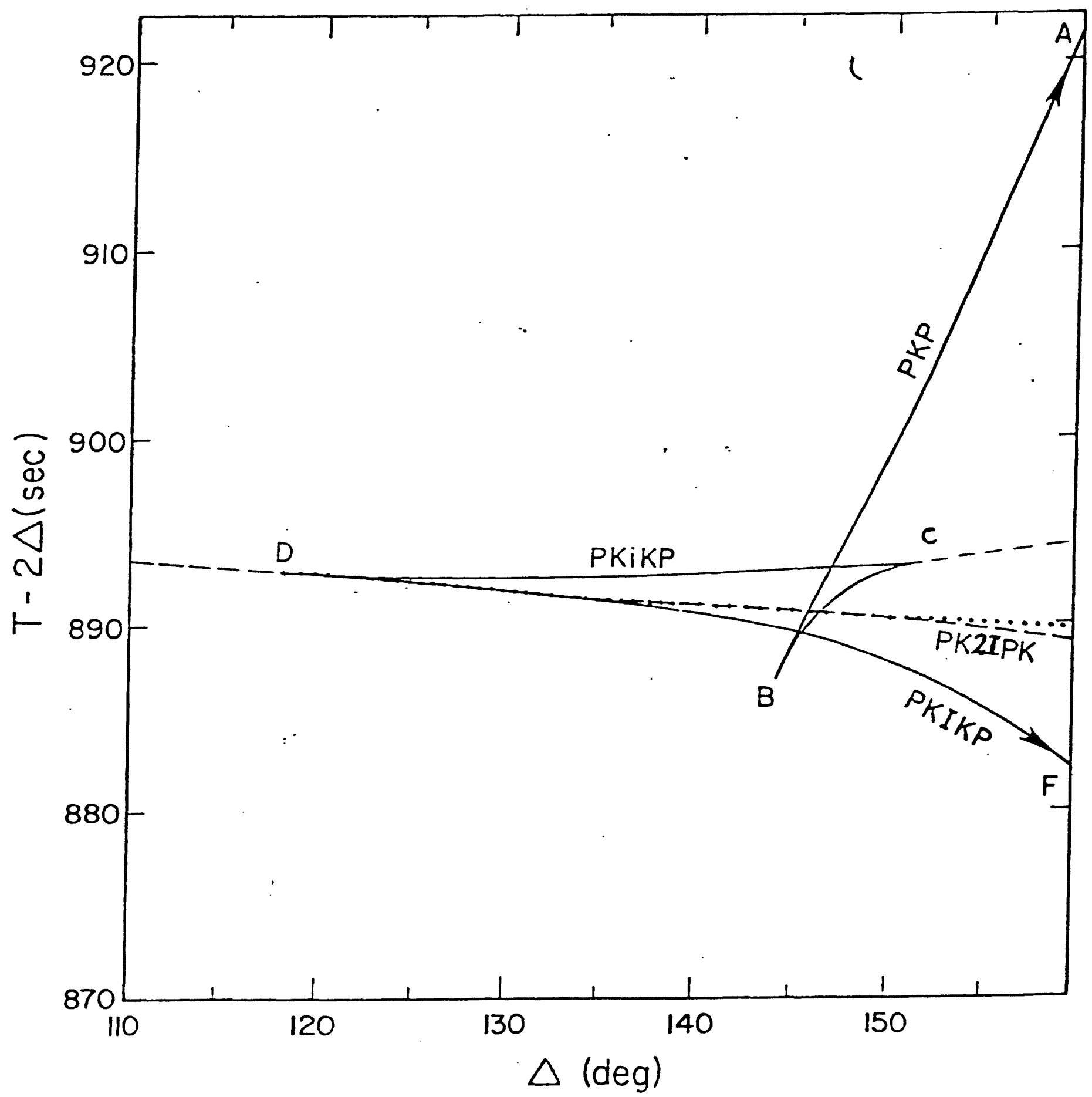

Figure 16. A reduced travel-time curve describing the PKP phases. The first few components of the interference head wave that emanates from the D-cusp are shown. It consists of the infinite sum of all the PKmIKP $(m=1,2, \ldots)$. The dashed extension to the $\mathrm{BC}$ branch describes the body wave that is diffracted beyond the $C$-cusp by propagating along the top of the inner core. 


\section{S-wave anisotropy}

There is currently support for putting anisotropy in portions of the upper mantle (e.g.. Dziewonski and Anderson, 1981). Such a feature is needed to resolve discrepancies in the S-velocity models derived from Rayleigh and Love wave data. However, instrumental limitations have until now made verification of anisotropy with body waves difficult. In order to observe anisotropy in Swaves, arrivals on horizontal channels must be rotated into radial and transverse components. This is a multichannel operation that is difficult to execute accurately with photographic records that must be converted to digital form. The problems encountered with hand-digitization (e.g., time-calibration. parallax of the recording instrument and skew of the amplitude-time axis caused by the helical recording) must be simultaneously corrected on the two horizontal components. If both long- and short-period components are used. then the multichannel operation requires exact time- and instrumentcalibrations over four different recordings from four different instruments. T. se problems are avoided for the most part at the GRF array. The data are recorded digitally by instruments with excellent calibration. The broadband response also means that one channel contains all the spectral information formerly split by the WWSSN system into long- and short-period recordings.

An example of how anisotrcpy can be easily detected with digital data is shown in Figs. 17-18. Fig. 17 shows the S-wave from a deep earthquake in the Kuriles as recorded on the three-component seismographs at the A1 and B1 stations. No discernible difference in arrival time can be measured between the $\mathrm{N}$ and $\mathrm{E}$ components of the raw records at either station (Fig. 17). (Note that the polarity of the horizontals at the B1 station were reversed. This in no way affects arrival time.) Figs. $18 \mathrm{a}$ and $18 \mathrm{~b}$ show the rotated components of the A1 and $\mathrm{B} 1$ channels respectively. It is clear, especially in the $\mathrm{A} 1$ records, that the transverse (SH) phase arrives before the radial (SV) phase. The time difference 

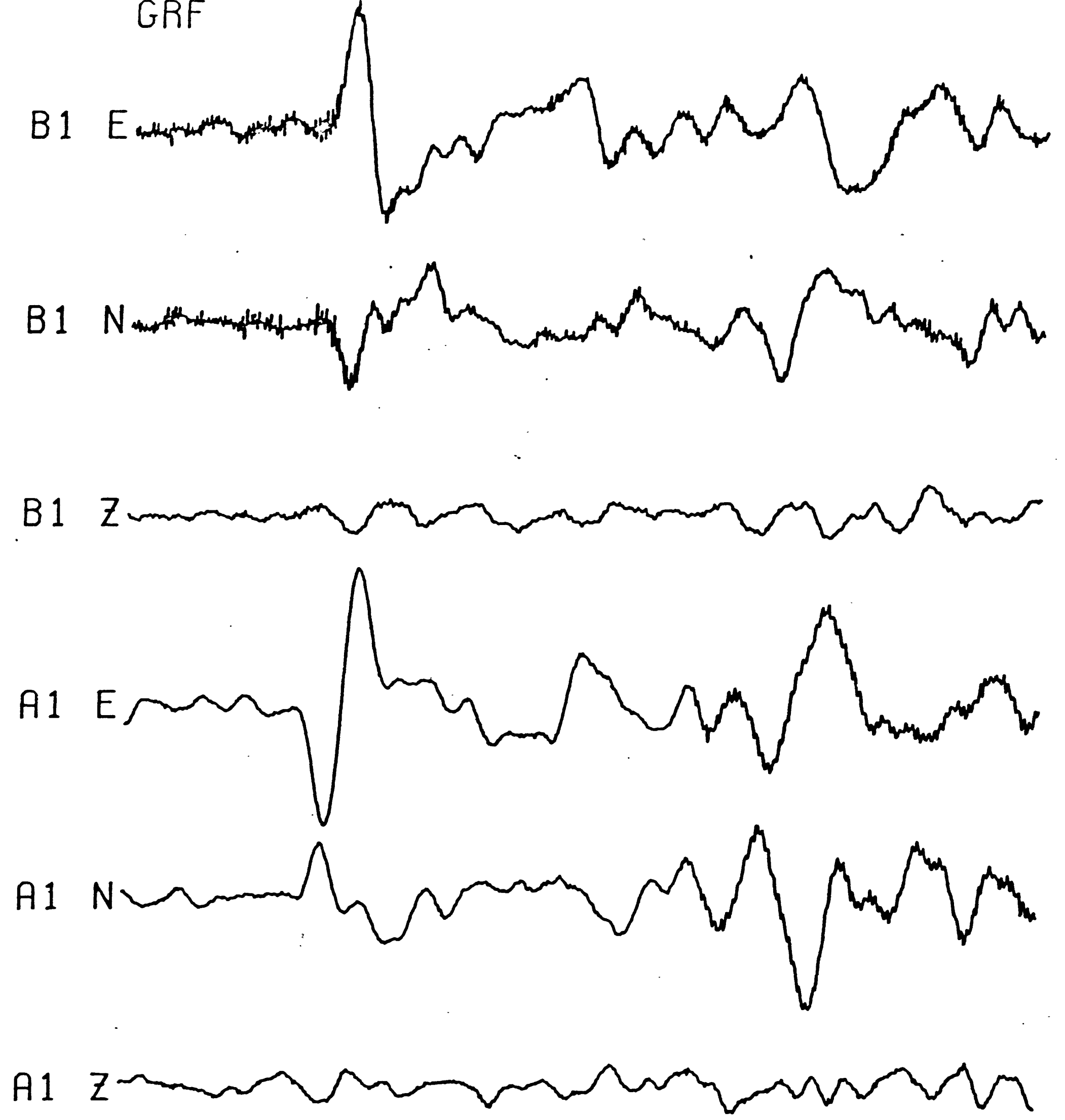

$11: 30: 40$

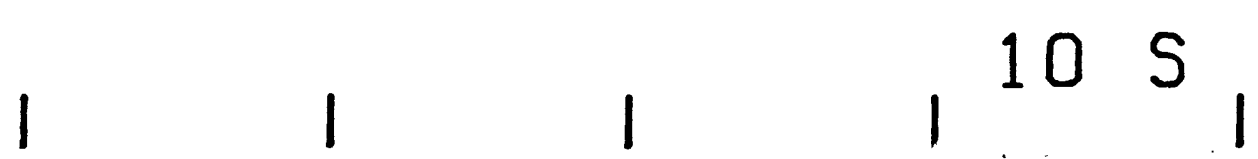

Figure 17. Broadband S-wave velocity waveforms as recorded by the threecomponent. Wielandt sensors at the GRF array for the deep earthquake from horizontals at the $\mathrm{B} 1$ station were reversed. This polarity reversal was corrected when these records were later used to form radial and transverse traces. There appears to be no travel-time differences between arrivals on the $\mathrm{N}$ and $\mathrm{E}$ components. 


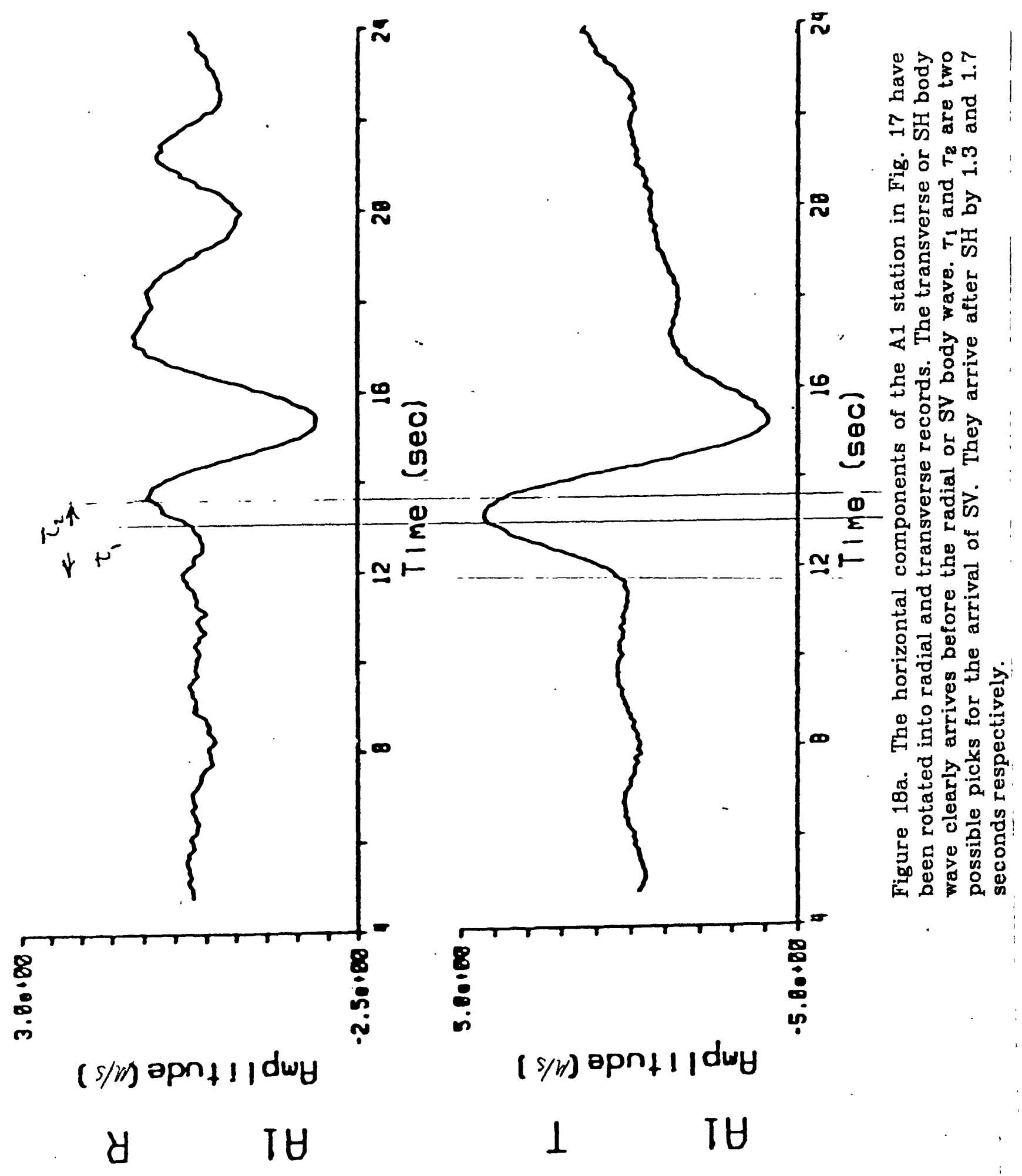



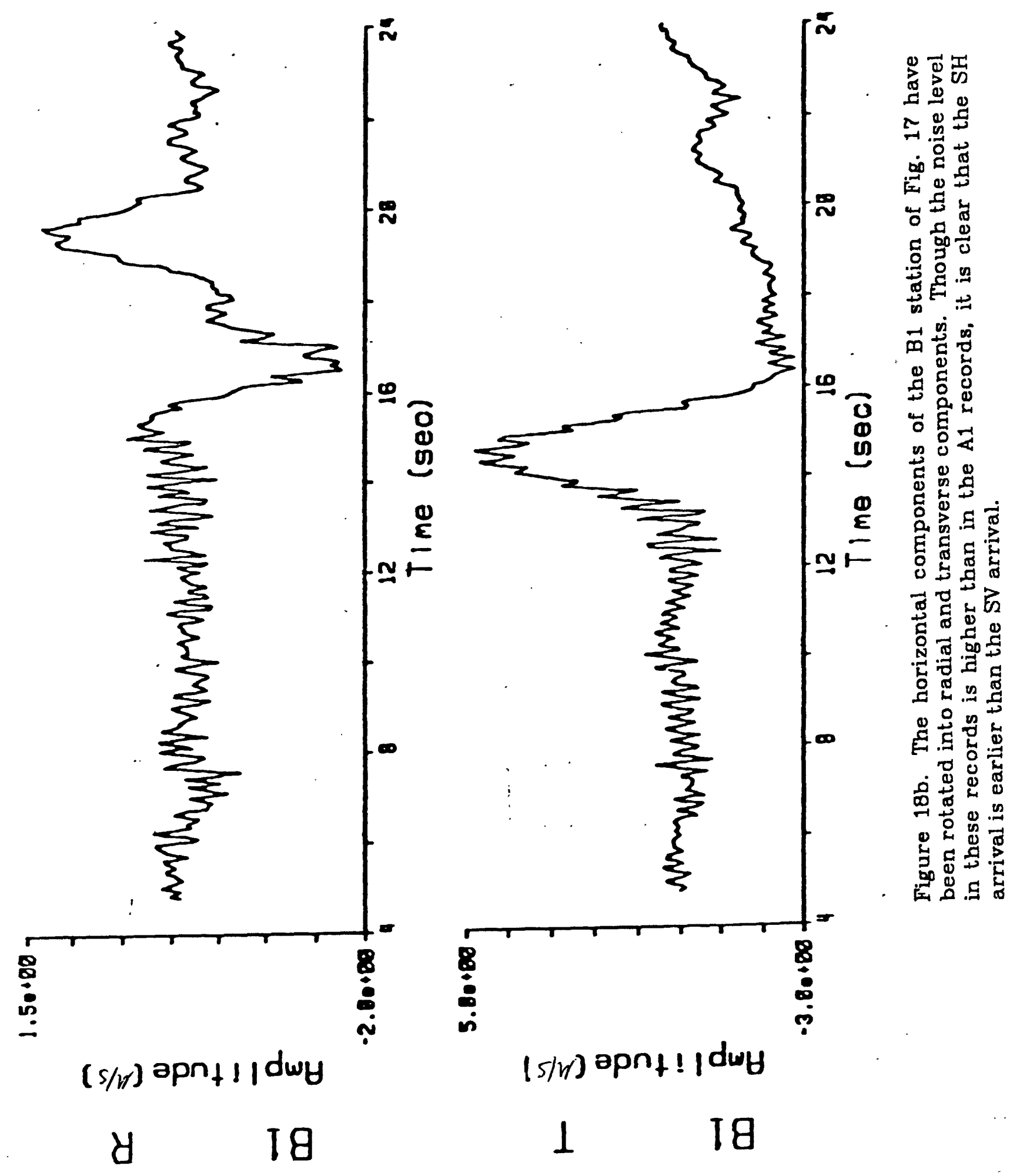
is between 1.3-1.7 seconds, depending on which feature of the radial record is chosen as the arrival time. This differential time is a minimum, because if an Sto-P conversion occurs beneath the station, it would arrive before SV. Yet the $\mathrm{SH}$ arrival is clearly earlier than anything above the noise level in the radial record.

Note that the SRO instrument has the same capability for detecting anisotropy as the Wielandt seismometer used in the GRF array. It also records digitally with a broadband capability. However, at this time the horizontal channels only record a filtered long-period output which may be insufficient to resolve the differential times that are predicted by anisotropy.

If the upper-mantle structure which causes S-wave anisotropy can be successfully modelled, then the anisotropic effects on P-waves can be estimated. It may turn out that the effects contribute to a significant part of travel-time residuals. In that case, a correction for anisotropy would lead to improved source locations as well as an improved gross earth model.

\section{ACKNOWLEDGMENTS}

This work was funded by the Defense Advanced Research Projects Agency. The author wishes to express his gratitude to Dr. Rainer Kind for providing invaluable assistance during my visit to the Graefenberg Seismological Observatory, and to Madeleine Zirbes for her help in processing data brought back from the observatory. 


\section{REFERENCES}

Choy, G. L., and J. Boatwright, 1981. The rupture characteristics of two deep earthquakes inferred from broadband GDSN data, Bull. Seism. Soc. Am, 71, 691-711.

Choy, G. L., and V. F. Cormier, 1981. The structure of the inner core inferred from short-period and broadband GDSN data, submitted to Geophys. J. $R$. astr. Soc.

Cormier, V. F. and G. L. Choy, 1981. Theoretical body wave interactions with upper mantle structure, J. Geophys. Res., 66, 1673-1678.

Dziewonski, A. and D. L. Anderson, 1981. Preliminary reference earth model, Phys. Earth Planet. Int., 25, 297-358.

Harvey, D. and G. L. Choy, 1981. Broadband deconvolution of GDSN data, Geophys. J. R. astr. Soc., in press.

Harjes, H.-P. and D. Seidl, 1978. Digital recording and analysis of broadband seismic data at the Graefenberg(GRF)-array, J. Geophys., 44, 511-523.

Seidl, D., 1980. The simulation problem for broad-band seismograms, $J$. Geophys., 48, 84-93. 